\title{
A Minimax Tracking Design for Wheeled Vehicles with Trailer Based on Adaptive Fuzzy Elimination Scheme
}

\author{
Bor-Sen Chen, Senior Member, IEEE, Charng-Shi Wu, and Huey-Jian Uang
}

\begin{abstract}
A robust tracking control design for wheeled vehicle systems with trailer, based on adaptive fuzzy elimination technique, is proposed in this study. A minimax control equipped with adaptive fuzzy elimination scheme is used to achieve a robust tracking performance, despite the system uncertainties and external disturbance. The design procedure is divided into three steps. First, a nominal wheeled vehicle control design is obtained via model reference tracking with desired eigenvalue assignment. Second, a fuzzy logic system is constructed, which is subsequently tuned to eliminate the nonlinear uncertainties as much as possible, in order to enhance the tracking robustness. Finally, a minimax control scheme is specified to optimally attenuate the worst case effect of the residue of fuzzy elimination on tracking error below a desired level to meet a robust tracking performance. An adaptive fuzzy-based $H_{\infty}$ optimization theory has been employed to solve the minimax problem. A simulation experiment of the wheeled vehicle with trailer is also presented in order to illustrate the tracking performance of the proposed design method.
\end{abstract}

Index Terms-Adaptive fuzzy elimination, $H_{\infty}$ control, minimax control, robust tracking control, wheeled vehicle.

\section{INTRODUCTION}

$\mathbf{T}$ HE growing use of wheeled vehicles with trailer in numerous applications, such as transportation vehicles, surveillance and ferry mobile robots, necessitates development of strategies for auto-parking, docking as well as path tracking and correction in higher speeds, in both structured as well as unstructured environments. The dynamics of the wheeled vehicle with trailer is one kind of nonholonomic systems.

During the last five years, the published literature pertaining to the nonholonomic control problem has grown enormously. In [1], an invariant manifold technique and a control strategy has been proposed which steers the nonholonomic systems onto manifolds of global and relative local-local controllability, to bring the nonholonomic vehicles under kinematic constraints. In [2], [3], the invariant manifold technique has been introduced to solve the tracking problem under dynamic and kinematic constraints. In [4], controllability of the general multibody mobile robotic system has been discussed. In [5], a summary of recent developments in control of nonholonomic systems, including tractors with trailers, robot manipulation, etc. has been presented. In [6], a control design for speed and azimuth of the

Manuscript received June 11, 1997; revised July 15, 1999. Recommended by Associate Editor, S. Farinwata. This work was supported by National Science Council under Contract NSC 88-2213-E-007-069.

The authors are with the Department of Electrical Engineering, National

Tsing Hua University, 30043 Hsin Chu, Taiwan.

Publisher Item Identifier S 1063-6536(00)03182-1. vehicles without trailer is solved by using the fuzzy-Gaussian neural-network controller. However, the parameter uncertainty and external disturbance have not been considered in this work, while the control performance can also not be guaranteed from a more theoretical perspective.

The present study concerns with the tracking control problem of a wheeled vehicle driven by two independent wheels. In the autonomous mobile robot system, an optimal control, or a model-based approach can be adopted for achieving such control, if the mathematical model is completely known. It is not easy, however, to measure some physical parameters, such as viscous friction factor and moment of inertia around the center of gravity (c.g.) for the wheeled vehicle., Therefore, a robust controller, not based on precise mathematical model, is recommendable for such control design since it allows simplification of modeling and also considers parameter variation, load change, elasticity of the wheels and road disturbance.

In the last decade, $H_{\infty}$ attenuation control design has been well developed for attenuating the effects of uncertain dynamics and external noises [13], [14]. However, if the magnitudes of uncertain dynamics and external noises are large, their effect on tracking error may still be obvious and even lead to system instability. In such situations, effective elimination of uncertain dynamics and external noise, prior to application of $H_{\infty}$ attenuation scheme, is necessary for enhancement of the tracking robustness. Conventionally, adaptive control schemes are employed to cancel the uncertain nonlinear dynamics. However, parameters are linear in conventional adaptive control schemes, i.e., the unknown parameters are considered to be of linear model form. In nonlinear uncertain wheeled vehicle systems, this may not be true. Since the fuzzy logic system can be tuned to approximate any nonlinear dynamic system, a fuzzy control technique has been used in this study to efficiently eliminate the plant uncertainties, via an adaptive learning method.

Recently, fuzzy techniques have been effectively used to approximate unknown nonlinear dynamics [7]-[9]. However, in the conventional adaptive fuzzy control, the desired cannot be guaranteed from a more theoretical control point of view. In this study, a fuzzy-based dynamic game scheme has been employed to treat robust tracking of uncertain wheeled vehicle systems. The proposed controller comprises of two terms, one being a self-tuning fuzzy logic system, which is employed to optimally eliminate the uncertain dynamics in order to enhance the tracking robustness, and the other being a minimax control algorithm, which is employed to attenuate the worst case effects of both the residue of fuzzy elimination and the exogenous dis- 
turbance on the tracking error within a desired level. The proposed design method attempts to combine the dynamic game technique and adaptive fuzzy algorithm to guarantee a robust tracking performance for uncertain wheeled vehicle control systems.

In the proposed robust tracking control, the adaptive fuzzy elimination technique is used as rough tuning while the minimax attenuation technique serves as fine tuning. Unlike the conventional adaptive control in nonlinear robust control systems, the uncertain dynamics need not be of linearly parameterized structure for this approach, i.e., uncertain dynamics can be free of structure in the proposed fuzzy-based robust control systems. Since the adaptive feedback linearization technique is avoided, an inverse of adaptive matrix become unnecessary. Hence, computational complexity as well as sensitivity of control algorithm are significantly reduced. Therefore, the proposed design method is more suitable for robust tracking performance control design for wheeled vehicle systems with uncertainties and external disturbance.

\section{Mathematical Model of the Wheeled Mobile Robot}

The following notations are used in this paper.

1) $I_{v}$ : moment of inertia around the c.g. of mobile robot.

2) $I_{w}$ : moment of inertia of the wheel.

3) $M_{1}$ : mass of the front wheeled vehicle.

4) $M_{2}$ : mass of the rear wheeled vehicle.

5) $l$ : distance between left or right wheel and c.g. of the wheeled vehicle.

6) $L$ : length of the link bar.

7) $d_{L}$ : distance between force $T$ and c.g. of the wheeled vehicle.

8) $\phi_{1}$ : azimuth of the front wheeled vehicle.

9) $\phi_{2}$ : azimuth of the rear wheeled vehicle.

10) $\left(x_{1}, y_{1}\right)$ : position of the front wheeled vehicle in absolute coordinate $O-X Y$.

11) $\left(x_{2}, y_{2}\right)$ : position of the rear wheeled vehicle in absolute coordinate $O-X Y$.

12) $v_{1}$ : velocity of the front wheeled vehicle.

13) $v_{2}$ : velocity of the rear wheeled vehicle.

14) $r$ : radius of the wheel.

15) $\theta_{l}, \theta_{r}$ : rotational angles of the left and right wheel, respectively.

16) $c$ : viscous friction factor.

17) $k$ : driving gain factor.

18) $u_{l}, u_{r}$ : driving input.

19) $T$ : tension force acting on the rear mobile vehicle.

20) $D_{l}, D_{r}$ : left and right driving forces.

Let the wheeled vehicle with two independent driving wheels be rigid, moving on the plane as shown in Fig. 1, where $O-X Y$ indicates the absolute (fixed) coordinates on the plane. In this paper, we deal with the wheeled vehicle under the following assumptions.

\section{Assumptions 1:}

1) The running wheeled vehicle satisfies both the pure rolling and nonslipping conditions.

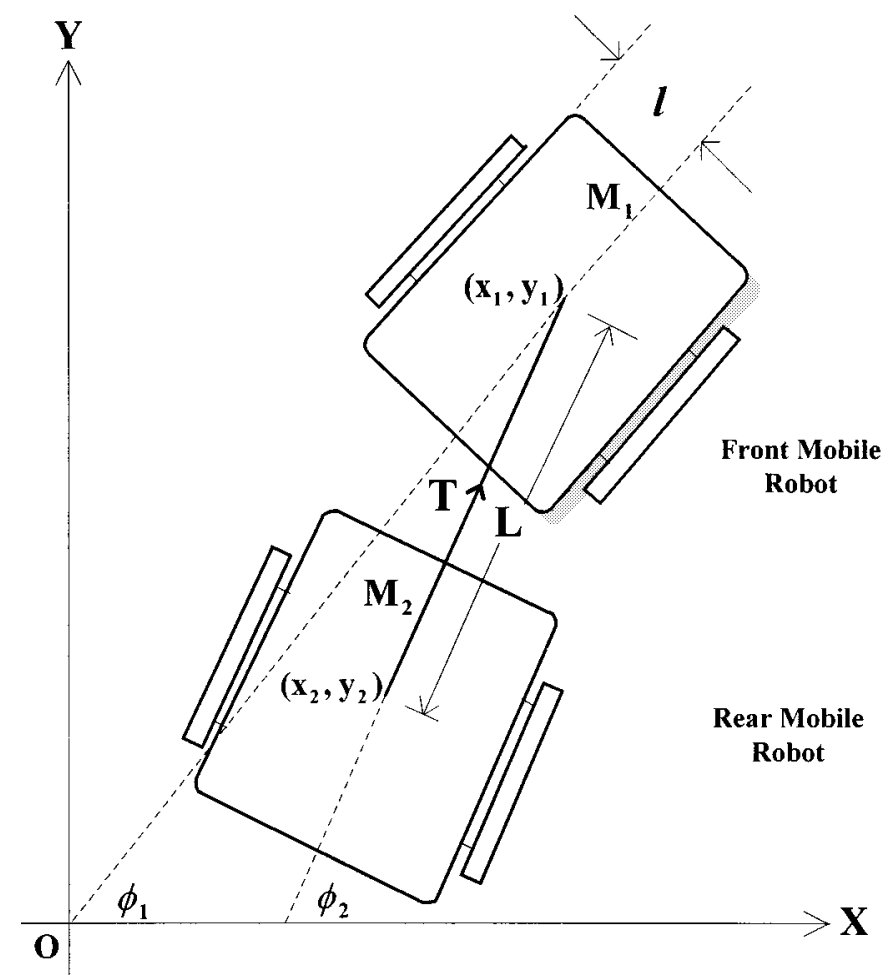

Fig. 1. A wheeled mobile robot with trailer and two independent wheels.

2) The link bar of the wheeled vehicle crosses the centers of gravity of the front and rear wheeled vehicles, i.e., $d_{L}=$ 0 .

Under the above assumptions, the model of such wheeled vehicle can be composed of the following equations [2], [6].

1) The dynamic equation of the front mobile robot

$$
\begin{aligned}
I_{v} \ddot{\phi}_{1}-T d_{L} & =D_{r} l-D_{l} l \\
M_{1} \dot{v}_{1}-T \cos \left(\phi_{2}-\phi_{1}\right) & =D_{r}+D_{l} .
\end{aligned}
$$

2) The dynamic equation of the driving systems for right and left wheels of the front mobile robot

$$
\begin{gathered}
I_{w} \ddot{\theta}_{r}+\dot{\theta}_{r}=k u_{r}-r D_{r} \\
I_{w} \ddot{\theta}_{l}+\dot{\theta}_{l}=k u_{l}-r D_{l} .
\end{gathered}
$$

3) Pure rolling constraints

$$
r \dot{\theta}_{r}=v_{1}+l \dot{\phi}_{1}
$$

$$
r \dot{\theta}_{l}=v_{1}-l \dot{\phi}_{1} .
$$

4) Nonslipping constraints

$$
\dot{x}_{1} \sin \phi_{1}-\dot{y}_{1} \cos \phi_{1}=0
$$

$$
\dot{x}_{2} \sin \phi_{2}-\dot{y}_{2} \cos \phi_{2}=0
$$


or

$$
\begin{aligned}
& \dot{x}_{1}=v_{1} \cos \phi_{1} \\
& \dot{y}_{1}=v_{1} \sin \phi_{1} \\
& \dot{x}_{2}=v_{2} \cos \phi_{2} \\
& \dot{y}_{2}=v_{2} \sin \phi_{2} .
\end{aligned}
$$

5) Relationships between front and rear wheeled vehicles

$$
\begin{aligned}
& T=M_{2} \dot{v}_{2} \\
& v_{2}=v_{1} \cos \left(\phi_{2}-\phi_{1}\right) \\
& \dot{\phi}_{2}=-\frac{1}{L} v_{1} \sin \left(\phi_{2}-\phi_{1}\right) .
\end{aligned}
$$

For the convenience of analysis, we combine the above equations in the following. First, substituting (4) and (5) into (2) and (3), we get

$$
\begin{aligned}
& \frac{I_{w}}{r}\left(\dot{v}_{1}+l \ddot{\phi}_{1}\right)+\frac{c}{r}\left(v_{1}+l \dot{\phi}_{1}\right)=k u_{r}-r D_{r} \\
& \frac{I_{w}}{r}\left(\dot{v}_{1}+l \ddot{\phi}_{1}\right)+\frac{c}{r}\left(v_{1}-l \dot{\phi}_{1}\right)=k u_{l}-r D_{l} .
\end{aligned}
$$

From (1) and (12)-(14), we obtain

$$
\begin{aligned}
& D_{r}=\frac{\left[l M_{1} \dot{v}_{1}-l T \cos \left(\phi_{2}-\phi_{1}\right)+I_{v} \ddot{\phi}_{1}\right]}{2 l} \\
& D_{r}=\frac{\left[l M_{1} \dot{v}_{1}-l T \cos \left(\phi_{2}-\phi_{1}\right)-I_{v} \ddot{\phi}_{1}\right]}{2 l}
\end{aligned}
$$

and

$$
\begin{aligned}
T= & {\left[M_{2} \cos \left(\phi_{2}-\phi_{1}\right) \quad 0\right]\left[\begin{array}{c}
\dot{v}_{1} \\
\ddot{\phi}_{1}
\end{array}\right] } \\
& +\left[\frac{1}{L} M_{2} v_{1} \sin ^{2}\left(\phi_{2}-\phi_{1}\right) M_{2} v_{1} \sin \left(\phi_{2}-\phi_{1}\right)\right] \\
& \times\left[\begin{array}{c}
v_{1} \\
\dot{\phi}_{1}
\end{array}\right]
\end{aligned}
$$

respectively. Substituting (17), (18) and (19) into (15) and (16), we get the following differential equation:

$$
M\left(\phi_{1}, \phi_{2}\right)\left[\begin{array}{c}
\dot{v}_{1} \\
\ddot{\phi}
\end{array}\right]+C\left(v_{1}, \phi_{1}, \phi_{2}\right)\left[\begin{array}{c}
v_{1} \\
\dot{\phi}_{1}
\end{array}\right]=\left[\begin{array}{l}
u_{r} \\
u_{l}
\end{array}\right]
$$

where

$$
\begin{aligned}
& M\left(\phi_{1}, \phi_{2}\right) \\
& \quad=\left[\begin{array}{ll}
\frac{I_{w}}{k r}+\frac{r M_{1}}{2 k}-\frac{r M_{2}}{2 k} \cos ^{2}\left(\phi_{2}-\phi_{1}\right) & \frac{I_{w} l}{k r}+\frac{r I_{V}}{2 l k} \\
\frac{I_{w}}{k r}+\frac{r M_{1}}{2 k}-\frac{r M_{2}}{2 k} \cos ^{2}\left(\phi_{2}-\phi_{1}\right) & \frac{I_{w l} l}{k r}-\frac{r I_{V}}{2 l k}
\end{array}\right]
\end{aligned}
$$

and $C\left(v_{1}, \phi_{1}, \phi_{2}\right)$ is shown at the bottom of the page.

Remark 1: For the wheeled vehicle system shown in Fig. 1, the position of the interaction through the link bar between the front wheeled vehicle and the rear wheeled vehicle has the following holonomic constraints in the $X-Y$ directions:

$$
x_{1}-x_{2}=L \cos \phi_{2}
$$

$$
y_{1}-y_{2}=L \sin \phi_{2} .
$$

Therefore, the trajectory planning for the wheeled vehicle systems must satisfy the requirements of the above constraints.

However, in practical control cases some parameter variations (or uncertainties) will occur on $I_{v}, I_{w}, M_{1}, M_{2}$ and $c$, i.e., these parameters can be composed of nominal and perturbed terms. In this situation, the coefficient matrices in (21) can be expressed as

$$
\begin{gathered}
M\left(\phi_{1}, \phi_{2}\right)=M_{0}\left(\phi_{1}, \phi_{2}\right)+\Delta M\left(\phi_{1}, \phi_{2}\right) \\
C\left(v_{1}, \phi_{1}, \phi_{2}\right)=C_{0}\left(v_{1}, \phi_{1}, \phi_{2}\right)+\Delta C\left(v_{1}, \phi_{1}, \phi_{2}\right)
\end{gathered}
$$

where the nominal coefficient matrices $M_{0}$ and $C_{0}$ are given by $M_{0}\left(\phi_{1}, \phi_{2}\right), C_{0}\left(v_{1}, \phi_{1}, \phi_{2}\right)$, shown at the bottom of the next page and the perturbed coefficient matrices $\Delta M$ and $\Delta C$ are given by $\Delta C\left(v_{1}, \phi_{1}, \phi_{2}\right)$, shown at the bottom of the next page.

$$
\begin{aligned}
& \Delta M\left(\phi_{1}, \phi_{2}\right) \\
& =\left[\begin{array}{ll}
\frac{\Delta I_{w}}{k r r}+\frac{r \Delta M_{1}}{2 k}-\frac{r \Delta M_{2}}{2 k} \cos ^{2}\left(\phi_{2}-\phi_{1}\right) & \frac{\Delta I_{w} l}{k r}+\frac{r \Delta I_{V}}{2 l k} \\
\frac{\Delta I_{w}}{k r}+\frac{r \Delta M_{1}}{2 k}-\frac{r \Delta M_{2}}{2 k} \cos ^{2}\left(\phi_{2}-\phi_{1}\right) & \frac{\Delta I_{w} l}{k r}-\frac{r \Delta I_{V}}{2 l k}
\end{array}\right] .
\end{aligned}
$$

Furthermore, external disturbances are also unavoidable. If $r_{1}$ denotes the position of the front wheeled vehicle, i.e., $\dot{r}_{1}=v_{1}$ and $d^{\prime}=\left[d_{1}^{\prime}, d_{2}^{\prime}\right]^{T}$ denotes the vector of external disturbance, then the equation of motion of the uncertain wheeled vehicle system can be expressed as

$$
\left(M_{0}+\Delta M\right) \ddot{q}+\left(C_{0}+\Delta C\right) \dot{q}=u+d^{\prime}
$$

where $q=\left[r_{1}, \phi_{1}\right]^{T}$ and $u=\left[u_{r}, u_{l}\right]^{T}$.

$$
C\left(v_{1}, \phi_{1}, \phi_{2}\right)=\left[\begin{array}{cc}
\frac{c}{k r}-\frac{r M_{2}}{2 k L} v_{1} \cos \left(\phi_{2}-\phi_{1}\right) \sin ^{2}\left(\phi_{2}-\phi_{1}\right) & \frac{c l}{k r}-\frac{r M_{2}}{2 k} v_{1} \cos \left(\phi_{2}-\phi_{1}\right) \sin \left(\phi_{2}-\phi_{1}\right) \\
\frac{c}{k r}-\frac{r M_{2}}{2 k L} v_{1} \cos \left(\phi_{2}-\phi_{1}\right) \sin ^{2}\left(\phi_{2}-\phi_{1}\right) & -\frac{c l}{k r}-\frac{r M_{2}}{2 k} v_{1} \cos \left(\phi_{2}-\phi_{1}\right) \sin \left(\phi_{2}-\phi_{1}\right)
\end{array}\right] .
$$


Let us consider the following nominal control law

$$
u=M_{0}\left[\ddot{q}_{d}-K_{1} \tilde{q}-K_{2} \dot{\tilde{q}}+u_{0}\right]+C_{0} \dot{q}
$$

where $K_{1}$ and $K_{2}$ are diagonal matrices to be designed, $u_{0}$ is an auxiliary control signal to be specified, $q_{d}=\left[\begin{array}{ll}r_{1 d} & \phi_{1 d}\end{array}\right]^{T}$ denotes the desired trajectory vector of the wheeled vehicle and $\tilde{q}$ is defined as

$$
\tilde{q} \triangleq q-q_{d}=\left[\begin{array}{c}
r_{1}-r_{1 d} \\
\phi_{1}-\phi_{1 d}
\end{array}\right]
$$

By substituting (27) into (26), we get

$$
M_{0}\left(\ddot{\tilde{q}}+K_{1} \tilde{q}+K_{2} \dot{\tilde{q}}\right)+\Delta M \ddot{q}+\Delta C \dot{q}=M_{0} u_{0}+d^{\prime}
$$

or

$$
\begin{aligned}
& \ddot{\tilde{q}}+K_{1} \tilde{q}+K_{2} \dot{\tilde{q}}+M_{0}^{-1}(\Delta M \ddot{q}+\Delta C \dot{q}) \\
& \quad=u_{0}+M_{0}^{-1} d^{\prime} .
\end{aligned}
$$

If we define

$$
e=\left[\begin{array}{c}
\tilde{q} \\
\tilde{q}
\end{array}\right]=\left[\begin{array}{c}
r_{1}-r_{1 d} \\
\phi_{1}-\phi_{1 d} \\
\dot{r}_{1}-\dot{r}_{1 d} \\
\dot{\phi}_{1}-\dot{\phi}_{1 d}
\end{array}\right]
$$

and

$$
\begin{aligned}
& f(\eta)=-M_{0}^{-1}(\Delta M \ddot{q}+\Delta C \dot{q}) \\
& d(t)=M_{0}^{-1} d^{\prime}(t)
\end{aligned}
$$

with $\eta=(\dot{q}, \ddot{q})^{T}$, then we get the tracking error dynamic equation of the wheeled vehicle system as follows:

$$
\dot{e}(t)=A e(t)+B\left(u_{0}+f(\eta)+d(t)\right) .
$$

where

$$
A=\left[\begin{array}{cc}
O_{2 \times 2} & I_{2 \times 2} \\
-K_{1} & -K_{2}
\end{array}\right]
$$

$$
\begin{aligned}
& =\left[\begin{array}{cccc}
0 & 0 & 1 & 0 \\
0 & 0 & 0 & 1 \\
-k_{11} & 0 & -k_{21} & 0 \\
0 & -k_{12} & 0 & -k_{22}
\end{array}\right] \\
B & =\left[\begin{array}{c}
O_{2 \times 2} \\
I_{2 \times 2}
\end{array}\right]=\left[\begin{array}{cc}
0 & 0 \\
0 & 0 \\
1 & 0 \\
0 & 1
\end{array}\right] .
\end{aligned}
$$

Assumption 2: The parameter variations $\Delta I_{v}, \Delta I_{w}, \Delta M_{1}$, $\Delta M_{2}$ and $\Delta c$ are bounded, then the induced norm of the perturbed coefficient matrices $\Delta M$ and $\Delta C$ in (24) and (25), and $f(\eta)$ in (32) are bounded also.

\section{Remarks 2:}

1) Note that the feedback control law (27) for the nonlinear system (26) is obtained via the nominal parameter matrices $M_{0}$ and $C_{0}$. It leads to the tracking error dynamic equation (34).

2) The control parameters $k_{11}, k_{12}, k_{21}$ and $k_{22}$ in (34) are specified so that all eigenvalues of $A$ have their desired values. Thus, by setting $u_{0}=0$, the tracking error $e(t)$ of dynamic equation (34) will approach zero as $t \rightarrow \infty$ when the wheeled vehicle system is free of uncertainty $f(\eta)$ and external disturbance $d(t)$.

\section{A BRIEF REVIEW OF FUZZY LOGIC SySTEM AND PROBLEM DESCRIPTION}

The tracking error dynamics of the uncertain wheeled vehicle is described by (34). If the wheeled vehicle is free of uncertainties and external disturbances (i.e., $f(\eta)=0$ and $d(t)=0$ ), the tracking error $e(t)$ in (34) will asymptotically converge to zero as $t \rightarrow \infty$ owing to the nonlinear control law (27), with adequate choice of linear control $u_{0}(t)$. In practical wheeled vehicle systems, however, uncertainties due to parameter perturbations, unmodeled dynamics, elasticity of the wheels, change of load and external disturbance of air resistance are unavoidable. Hence, it is important to eliminate the effect of uncertainties $f(\eta)$ and $d(t)$ on tracking error in (34). Since $f(\eta)$ is uncertain, several robust design algorithms [13]-[17] can be employed as robust controllers to override the upper norm bound of $\|f(\eta)\|$. Due to high nonlinearity and uncertainty of $f(\eta)$, it is, however, not easy to estimate the upper norm bound. Furthermore, it is also

$$
\begin{aligned}
M_{0}\left(\phi_{1}, \phi_{2}\right)= & {\left[\begin{array}{ll}
\frac{I_{w 0}}{k r}+\frac{r M_{10}}{2 k}-\frac{r M_{20}}{2 k} \cos ^{2}\left(\phi_{2}-\phi_{1}\right) & \frac{I_{w 0} l}{k r}+\frac{r I_{V 0}}{2 l k} \\
\frac{I w 0}{k r}+\frac{r M_{10}}{2 k}-\frac{r M_{20}}{2 k} \cos ^{2}\left(\phi_{2}-\phi_{1}\right) & \frac{I_{w 00} l}{k r}-\frac{r I_{V 0}}{2 l k}
\end{array}\right] } \\
C_{0}\left(v_{1}, \phi_{1}, \phi_{2}\right)= & {\left[\begin{array}{ll}
\frac{c_{0}}{k r}-\frac{r M_{20}}{2 k L} v_{1} \cos \left(\phi_{2}-\phi_{1}\right) \sin ^{2}\left(\phi_{2}-\phi_{1}\right) & \frac{c_{0} l}{k r}-\frac{r M_{20}}{2 k} v_{1} \cos \left(\phi_{2}-\phi_{1}\right) \sin \left(\phi_{2}-\phi_{1}\right) \\
\frac{c_{0}}{k r}-\frac{r M_{20}}{2 k L} v_{1} \cos \left(\phi_{2}-\phi_{1}\right) \sin ^{2}\left(\phi_{2}-\phi_{1}\right) & -\frac{c_{0} l}{k r}-\frac{r M_{20}}{2 k} v_{1} \cos \left(\phi_{2}-\phi_{1}\right) \sin \left(\phi_{2}-\phi_{1}\right)
\end{array}\right] }
\end{aligned}
$$

$$
\Delta C\left(v_{1}, \phi_{1}, \phi_{2}\right)=\left[\begin{array}{lc}
\frac{\Delta c}{k r}-\frac{r \Delta M_{2}}{2 k L} v_{1} \cos \left(\phi_{2}-\phi_{1}\right) \sin ^{2}\left(\phi_{2}-\phi_{1}\right) & \frac{\Delta c l}{k r}-\frac{r \Delta M_{2}}{2 k} v_{1} \cos \left(\phi_{2}-\phi_{1}\right) \sin \left(\phi_{2}-\phi_{1}\right) \\
\frac{\Delta c}{k r}-\frac{r \Delta M_{2}}{2 k L} v_{1} \cos \left(\phi_{2}-\phi_{1}\right) \sin ^{2}\left(\phi_{2}-\phi_{1}\right) & -\frac{\Delta c l}{k r}-\frac{r \Delta M_{2}}{2 k} v_{1} \cos \left(\phi_{2}-\phi_{1}\right) \sin \left(\phi_{2}-\phi_{1}\right)
\end{array}\right]
$$




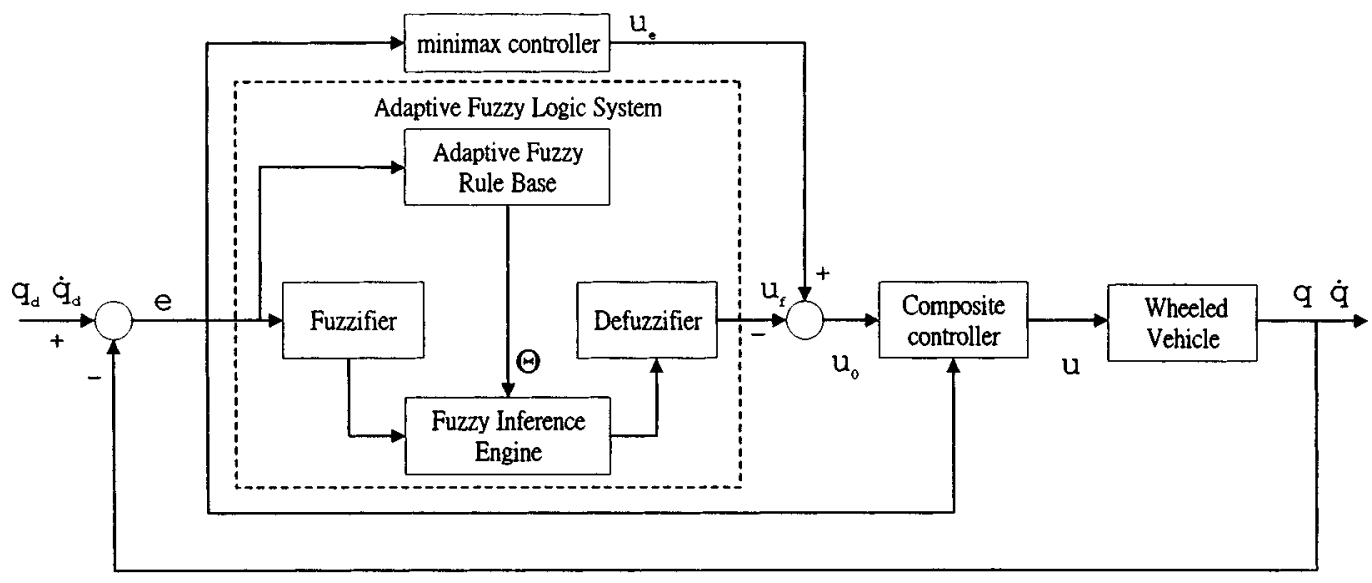

Fig. 2. Fuzzy logic system for wheeled vehicle tracking control.

not easy to suppress the effect of external disturbance $d(t)$ by using, for example, a VSS control or a dead-zone control based on the upper bound of external disturbance to treat this robust tracking problem. All of these robust control methods may lead to conservative and imprecise results.

In this paper, the control signal $u_{0}$ in (34) is divided into two parts as follows:

$$
u_{0}=u_{e}-u_{f}(\eta, \Theta)
$$

In this situation, the tracking error dynamics in (34) is of the following form:

$$
\dot{e}=A e+B u_{e}+B\left[f(\eta)-u_{f}(\eta, \Theta)\right]+B d
$$

where $u_{f}(\eta, \Theta)$ is a fuzzy logic system and $\Theta$ is the update parameter matrix which will be discussed in the following paragraph. In (36), the fuzzy logic system $u_{f}(\eta, \Theta)$ is tuned to approximate $f(\eta)$ as closely as possible. Furthermore, the control signal $u_{e}(t)$ is used to attenuate the effects of residue of $f(\eta)-u_{f}(\eta, \Theta)$ as well as the external disturbance $d(t)$ on the tracking error, from the minimax perspective.

The basic configuration of the fuzzy logic system for wheeled vehicle tracking control is shown in Fig. 2. The fuzzy logic system $u_{f}(\eta, \Theta)$ in this work performs a mapping from $U \in R^{4}$ to $V \in R^{2}$. Let $U=U_{1} \times U_{2} \times U_{3} \times U_{4}$ where $U_{i} \subset R$, for $i=1,2,3,4$. The fuzzy rule base consists of a collection of fuzzy If-Then rules as follows:

$$
\begin{aligned}
& R^{(l)}: \text { If } \eta_{1} \text { is } F_{1}^{l}, \eta_{2} \text { is } F_{2}^{l}, \eta_{3} \text { is } F_{3}^{l} \text { and } \eta_{4} \text { is } F_{4}^{l}, \\
& \text { Then } u_{f} \text { is } G^{l}, \quad \text { for } l=1,2, \ldots, M
\end{aligned}
$$

where $\eta=\left(\eta_{1}, \eta_{2}, \eta_{3}, \eta_{4}\right)^{T} \in U$ and $u_{f} \in V \subset R^{2}$ are the input and output of the fuzzy logic system, respectively. In our design, the fuzzy inference engine performs a mapping from fuzzy sets in $U \in R^{4}$ to fuzzy sets in $R^{4}$, based upon the fuzzy If-Then rules in the fuzzy rule base and the compositional rule of inference. The fuzzifier maps a crisp point $\eta=\left(\eta_{1}, \eta_{2}, \eta_{3}, \eta_{4}\right)^{T} \in U$ into a fuzzy set $A_{x}$ in $U$. The defuzzifier maps a fuzzy set in $V$ to a crisp point in $V$. More informations can be found in [18], [19] and [21].
The fuzzy logic system contained in Fig. 2 comprises a very rich class of static system mapping from $U \subset R^{4}$ to $V \subset R^{2}$, since many different choices are available within each block, and in addition, many combinations of these choices can result in a useful subclass of fuzzy logic systems. One subclass of fuzzy logic systems is used here as building blocks of the fuzzy approximation controller $u_{f}(\eta, \Theta)$ for adaptive cancellation of nonlinear function $f(\eta)$ and is described by the following important result.

Lemma [21]: If the fuzzy basis functions are defined as

$$
\xi_{i l}(\eta)=\frac{\prod_{j=1}^{4} \mu_{F_{j}^{i l}}\left(\eta_{j}\right)}{\sum_{k=1}^{M}\left[\prod_{j=1}^{4} \mu_{F_{j}^{i k}}\left(\eta_{j}\right)\right]}
$$

for $i=1,2$ and $l=1,2, \ldots, M$. Then the fuzzy logic systems with center-average defuzzifier, product inference and singleton fuzzifier for wheeled mobile robotic tracking systems, are of the following form [7]:

$$
u_{f}(\eta, \Theta)=\left[\begin{array}{l}
u_{f_{1}}(\eta, \Theta) \\
u_{f_{2}}(\eta, \Theta)
\end{array}\right]=\left[\begin{array}{l}
\xi_{1}^{T} \Theta_{1} \\
\xi_{2}^{T} \Theta_{2}
\end{array}\right]=\Xi(\eta) \Theta
$$

where

$$
\begin{aligned}
\Theta & =\left[\begin{array}{c}
\Theta_{1} \\
\Theta_{2}
\end{array}\right] \\
\Xi(\eta) & =\left[\begin{array}{cc}
\xi_{1}^{T}(\eta) & 0 \\
0 & \xi_{2}^{T}(\eta)
\end{array}\right]
\end{aligned}
$$

and $\Theta_{i}=\left[\begin{array}{llll}\theta_{i 1} & \cdots & \theta_{i M}\end{array}\right]^{T}, \xi_{i}(\eta)=\left[\begin{array}{llll}\xi_{i 1}(\eta) & \cdots & \xi_{i M}(\eta)\end{array}\right]^{T}$, for $i=1,2$ with that $\theta_{i l}$ is the point at which the given membership function $\mu_{F_{i}^{i l}}\left(\theta_{i l}\right)$ achieves its maximum value, and we assume that $\mu_{F_{j}^{i l}}\left(\theta_{i l}^{j}\right)=1$.

Remarks 3:

1) There are two main reasons for using the fuzzy logic system (39) as a basic building block of adaptive fuzzy controller. First, the fuzzy logic systems (39) are constructed from the fuzzy If-Then rules of (37), using some specific fuzzy inference, fuzzification, and defuzzification strategies. Therefore, linguistic information from a human expert can be directly incorporated into the controllers. Second, the fuzzy logic systems of the form (39) 
have been proven in [8] and [21] to be universal approximations, i.e., for any given real continuous function $f(\eta)$ defined on the compact set $U$, there exists a fuzzy logic system of the form (39) such that it can uniformly approximate $f(\eta)$ over $U$ to an arbitrary accuracy by adequate choice of parameter matrix $\Theta$ as $M \rightarrow \infty$. Therefore, the fuzzy logic systems (39) are qualified as building blocks for the adaptive controller for elimination of nonlinear uncertainties of wheeled vehicle systems.

2) In this design, the membership functions $\mu_{F^{i l}}$ are specified according to the designer's experience or knowledge about the uncertainties of the wheeled mobile systems and the parameter $\Theta$ is to be tuned according to the tracking error $e$.

However, in practical control design, the number of fuzzy basis functions of fuzzy logic system is chosen as small as possible for the convenience of computation and implementation. In this situation, an adaptive law must be developed to tune the parameter $\Theta$, in order to make a fuzzy logic system $u_{f}(\eta, \Theta)$ with adequate dimension to eliminate $f(\eta)$ as fully as possible.

Let us define the following optimal update parameter of elimination [21]:

$$
\Theta^{*}=\arg \min _{\Theta \in \Omega_{\Theta}} \max _{\eta \in \Omega_{\eta}}\|f(\eta)-\Xi(\eta) \Theta\|
$$

where $\|\cdot\|$ denotes the Euclidean norm, i.e., $\|\eta\|=\sqrt{\eta^{T} \eta}$ and $\Omega_{\Theta}$ and $\Omega_{\eta}$ denote the sets of $\Theta$ and $\eta$ that have suitable bounds, respectively. Obviously, $\Theta^{*}$ exists and is a constant. Furthermore, in $\Omega_{\Theta}$ and $\Omega_{\eta}, f(\eta)=\Xi(\eta) \Theta^{*}+\varepsilon(t)$ with $\varepsilon(t) \leq$ $\bar{\varepsilon}$ for some $\bar{\varepsilon}>0$. To guarantee that $\Theta$ lies inside the bounded set $\Omega_{\Theta}$, the projection algorithm is introduced to prevent the divergence of $\Theta$ in the next section.

Then, the tracking error dynamic equation (36) can be rewritten as follows:

$$
\begin{aligned}
\dot{e}= & A e+B u_{e}+B\left(\Xi(\eta) \Theta^{*}-\Xi(\eta) \Theta\right) \\
& +B \varepsilon(t)+B d \\
= & A e+B u_{e}+B \Xi(\eta) \tilde{\Theta}+B w
\end{aligned}
$$

where $w=\varepsilon(t)+d$ denotes the sum of optimal elimination error due to the fuzzy logic system and the external disturbance, and $\tilde{\Theta}(t)$ is defined as

$$
\tilde{\Theta}(t)=\Theta^{*}-\Theta(t) .
$$

The proposed design procedure is divided into two steps. In the first step, the adaptive fuzzy logic system $u_{f}(\eta, \Theta)$ is tuned by way of $\Theta(t)$ to optimally approximate and eliminate the uncertain term $f(\eta)$. In this situation, the term $\Xi(\eta) \Theta^{*}-\Xi(\eta) \Theta=$ $\Xi(\eta) \tilde{\Theta}$ vanishes as $t \rightarrow \infty$. However, the sum of approximation error and external disturbance $w$ can not be estimated and eliminated. Therefore, in the second step, the control signal $u_{e}(t)$ is specified such that the worst case effect of the uncertainty $w(t)$ on the tracking error $e(t)$ must be attenuated as much as possible, and below a prescribed level $\rho$, i.e., the following min- imax (optimal $H_{\infty}$ ) tracking performance must be satisfied for the error dynamics in (41):

$$
\begin{aligned}
& \min _{u_{e}(t) \in L_{2}\left[0, t_{f}\right]} \max _{w(t) \in L_{2}\left[0, t_{f}\right]} \int_{0}^{t_{f}}\left[e^{T}(t) Q e(t)\right. \\
& \left.+u_{e}^{T}(t) R u_{e}(t)-\rho^{2} w^{T}(t) w(t)\right] d t \\
& \leq e^{T}(0) P e(0)+\frac{1}{\gamma}\left[\tilde{\Theta}^{T}(0) \tilde{\Theta}(0)\right]
\end{aligned}
$$

where $\gamma>0$ is a weighting factor, $Q=Q^{T}>0, P=P^{T}>0$ and $R>0$ are some positive definite weighting matrices and the final time $t_{f}>0$ [10]-[13].

For the case $e(0)=0$ and $\tilde{\Theta}(0)=0,(43)$ is reduced to the following form [10]-[13]:

$$
\min _{u_{e}(t) \in L_{2}\left[0, t_{f}\right]} \max _{w(t) \in L_{2}\left[0, t_{f}\right]} \frac{\left\|\left[Q^{\frac{1}{2}} e(t) R^{\frac{1}{2}} u_{e}(t)\right]\right\|_{L_{2}}}{\|w(t)\|_{L_{2}}} \leq \rho
$$

where

$$
\begin{aligned}
\left\|\left[Q^{\frac{1}{2}} e(t) R^{\frac{1}{2}} u_{e}(t)\right]\right\|_{L_{2}} \\
\quad=\sqrt{\int_{0}^{t_{f}} e^{T}(t) Q e(t)+u_{e}^{T}(t) R u_{e}(t) d t}
\end{aligned}
$$

and

$$
\|w(t)\|_{L_{2}}=\sqrt{\int_{0}^{t_{f}} w^{T}(t) w(t) d t}
$$

However, in general, if $e(0) \neq 0$ and $\tilde{\Theta}(0) \neq 0$, the minimax performance in (44) must be modified to (43) by considering $e(0)$ and $\tilde{\Theta}(0)$ as some kind of disturbances [10], [11]. Our design objective is to tune the parameters of the fuzzy logic system to eliminate the uncertain term $\Xi(\eta) \Theta^{*}-\Xi(\eta) \Theta$, and then to derive an adequate minimax control law $u_{e}(t)$ so that the worst case effect of $w(t)$ on $e(t)$ is guaranteed to be less than or equal to $\rho$.

At present [10]-[13], the minimax control (or optimal $H_{\infty}$ control) is the most efficient method to eliminate the effect of uncertainty $w(t)$ on $e(t)$. Therefore, this method has been employed to treat the residue $w(t)$, and achieve robust tracking of the wheeled vehicle system by fuzzy-based minimax control design.

The expression (44) means that the worst case influence (induced $L_{2}$ norm) from $w(t)$ to $Q^{1 / 2} e(t)$ and $R^{1 / 2} u_{e}(t)$ must be minimized, and brought below a prescribed $\rho$. For achieving this, we are actually dealing with the minimax tracking problem for the model reference control of wheeled vehicle with robustness enhancement by using adaptive fuzzy logic system. The main difference between the proposed algorithm and the conventional $H_{\infty}$ control is that an adaptive fuzzy logic system $u_{f}(\eta, \Theta)$ is employed in the present design to eliminate the uncertain term $f(\eta)$ as fully as possible before the minimax control $u_{e}(t)$ is applied. In the conventional $H_{\infty}$ control design, only $H_{\infty}$ control $u_{e}(t)$ is employed to directly attenuate the uncertain terms $f(\eta)$ and $d(t)$. If $f(\eta)$ and $d(t)$ are very large, the 
stability may not be guaranteed. Even if the stability is guaranteed, the effect of $f(\eta)$ and $d(t)$ on tracking error will be still very large even if $H_{\infty}$ attenuation is used. Therefore, the result may be conservative and more control effort will be needed. The role of robustness enhancement via adaptive fuzzy logic system in the design of $H_{\infty}$ robust controller on the uncertain wheeled vehicle system will be discussed in the simulation example in Section IV.

Remarks 4:

1) In general, $\rho<1$ is necessary for the attenuation of the effect of $w(t)$ to achieve robust tracking. If $\rho \rightarrow \infty$ [10]-[13], then the robust $H_{\infty}$ tracking performance design is reduced to an $\mathrm{H}_{2}$ optimal tracking control design, without considering the attenuation of the effect of $w(t)$.

2) The performance in (43) or (44) is of finite time minimax tracking control. In this situation, $w(t)$ can be finite in $\left[0, t_{f}\right]$. If $t_{f} \rightarrow \infty$, the integrations $\int_{0}^{\infty}\left[e^{T}(t) Q e(t)+u_{e}^{T}(t) R u_{e}(t)\right] d t \quad$ and $\int_{0}^{\infty}\left[w^{T}(t) w(t)\right] d t$ may be increased to $\infty$. However, the integral inequality always holds.

\section{Minimax Tracking of Mobile Robot Systems Via ADAPTIVE FUZZY CANCELLATION SCHEME}

Based on the analysis in the above section, the robust tracking control design of uncertain wheeled vehicle systems is formulated as a fuzzy-based minimax tracking control design problem. An adaptive fuzzy control $u_{f}(\eta, \Theta)=\Xi(\eta) \Theta$ is used to eliminate the uncertainty $f(\eta)$ as fully as possible and then a minimax control $u_{e}$ is specified to attenuate the worst case influence of $w(t)$ on tracking error below a prescribed level $\rho$. The main work in this design is to specify an update law for $\Theta$ and a control law $u_{e}(t)$ such that for any $w(t)$ the minimax performance (43) or (44) is achieved.

Subject to the tracking error dynamic equation (41), let es define the cost functional

$$
\begin{aligned}
J\left(e, u_{e}, w\right)= & \int_{0}^{t_{f}}\left[e^{T}(t) Q e(t)+u_{e}^{T}(t) R u_{e}(t)\right. \\
& \left.-\rho^{2} w^{T}(t) w(t)\right] d t .
\end{aligned}
$$

Then we get the following main result.

Theorem: For the uncertain wheeled vehicle system (26), if the control is chosen as

$$
u(t)=M_{0}\left(\ddot{q}_{d}-K_{1} \tilde{q}-K_{2} \dot{\tilde{q}}+u_{e}-\Xi \Theta\right)+C_{0} \dot{q}
$$

with

$$
\begin{aligned}
& \dot{\Theta}=\gamma \Xi^{T}(\eta) B^{T} P e(t) \\
& u_{e}=-R^{-1} B^{T} P e(t)
\end{aligned}
$$

where $R=R^{T}$ is a weighting matrix and $P=P^{T}>0$ is the solution of the following algebraic Riccati-like equation:

$$
P A+A^{T} P+Q-P B\left(R^{-1}-\frac{1}{\rho^{2}} I\right) B^{T} P=0 .
$$

Then the minimax tracking performance in (43) is guaranteed for a prescribed $\rho$ and the corresponding worst case $w^{*}(t)$ deteriorating the tracking performance is of the form

$$
w^{*}(t)=\frac{1}{\rho^{2}} B^{T} P e(t) .
$$

Proof: By the fact that

$$
\begin{aligned}
& e^{T}(0) P e(0)-e^{T}\left(t_{f}\right) P e\left(t_{f}\right)+\frac{1}{\gamma} \tilde{\Theta}^{T}(0) \tilde{\Theta}(0) \\
& -\frac{1}{\gamma} \tilde{\Theta}^{T}\left(t_{f}\right) \tilde{\Theta}\left(t_{f}\right)+\int_{0}^{t_{f}}\left[\dot{e}^{T}(t) P e(t)+e^{T}(t) P \dot{e}(t)\right. \\
& \left.+\frac{1}{\gamma} \dot{\tilde{\Theta}}^{T}(t) \tilde{\Theta}(t)+\frac{1}{\gamma} \tilde{\Theta}^{T}(t) \dot{\tilde{\Theta}}(t)\right] d t \\
& \quad=0
\end{aligned}
$$

Eq. (45) is equivalent to

$$
\begin{aligned}
& J\left(e, u_{e}, w\right) \\
&= e^{T}(0) P e(0)-e^{T}\left(t_{f}\right) P e\left(t_{f}\right)+\frac{1}{\gamma} \tilde{\Theta}^{T}(0) \tilde{\Theta}(0) \\
&-\frac{1}{\gamma} \tilde{\Theta}^{T}\left(t_{f}\right) \tilde{\Theta}\left(t_{f}\right)+\int_{0}^{t_{f}}\left[e^{T}(t) Q e(t)+u_{e}^{T}(t) R u_{e}(t)\right. \\
&-\rho^{2} w^{T}(t) w(t)+\dot{e}^{T}(t) P e(t)+e^{T}(t) P \dot{e}(t) \\
&\left.+\frac{1}{\gamma} \dot{\tilde{\Theta}}^{T}(t) \tilde{\Theta}(t)+\frac{1}{\gamma} \tilde{\Theta}^{T}(t) \dot{\tilde{\Theta}}(t)\right] d t .
\end{aligned}
$$

Substituting (41) into the above equation, we get

$$
\begin{aligned}
& J\left(e, u_{e}, w\right) \\
& =e^{T}(0) P e(0)-e^{T}\left(t_{f}\right) P e\left(t_{f}\right)+\frac{1}{\gamma} \tilde{\Theta}^{T}(0) \tilde{\Theta}(0) \\
& -\frac{1}{\gamma} \tilde{\Theta}^{T}\left(t_{f}\right) \tilde{\Theta}\left(t_{f}\right) \\
& +\int_{0}^{t_{f}}\left[e^{T}(t)\left(A^{T} P+P A+Q\right) e(t)\right. \\
& \quad+u_{e}^{T}(t) R u_{e}(t)-\rho^{2} w^{T}(t) w(t)+u_{e}^{T}(t) B^{T} P e(t) \\
& \quad+e^{T}(t) P B u_{e}(t)+\tilde{\Theta}^{T}(t) \Xi^{T}(\eta) B^{T} P e(t) \\
& \quad+e^{T}(t) P B \Xi(\eta) \tilde{\Theta}(t)+w^{T}(t) B^{T} P e(t) \\
& \quad+e^{T}(t) P B w(t)+\frac{1}{\gamma} \dot{\tilde{\Theta}}(t) \tilde{\Theta}(t) \\
& \left.\quad+\frac{1}{\gamma} \tilde{\Theta}^{T}(t) \dot{\tilde{\Theta}}(t)\right] d t .
\end{aligned}
$$

By the update law in (47) and the fact that $\dot{\tilde{\Theta}}=-\dot{\Theta}$ from (42), (52) can be rewritten as

$$
\begin{aligned}
& J\left(e, u_{e}, w\right) \\
&= e^{T}(0) P e(0)+\frac{1}{\gamma} \tilde{\Theta}^{T}(0) \tilde{\Theta}(0)-e^{T}\left(t_{f}\right) P e\left(t_{f}\right) \\
&-\frac{1}{\gamma} \tilde{\Theta}^{T}\left(t_{f}\right) \tilde{\Theta}\left(t_{f}\right) \\
&+\int_{0}^{t_{f}}\left[e^{T}(t)\left(A^{T} P+P A+Q\right) e(t)\right. \\
&+u_{e}^{T}(t) R u_{e}(t)-\rho^{2} w^{T}(t) w(t)+u_{e}^{T}(t) B^{T} P e(t) \\
&+w^{T}(t) B^{T} P e(t)+e^{T}(t) P B u_{e}(t) \\
&\left.+e^{T}(t) P B w(t)\right] d t .
\end{aligned}
$$


Using (49) and applying the technique of completing the squares, we get

$$
\begin{aligned}
& J\left(e, u_{e}, w\right) \\
&=e^{T}(0) P e(0)+\frac{1}{\gamma} \tilde{\Theta}^{T}(0) \tilde{\Theta}(0)-e^{T}\left(t_{f}\right) P e\left(t_{f}\right) \\
&-\frac{1}{\gamma} \tilde{\Theta}^{T}\left(t_{f}\right) \tilde{\Theta}\left(t_{f}\right)+\int_{0}^{t_{f}} {\left[\left(R u_{e}(t)+B^{T} P e(t)\right)^{T}\right.} \\
& \times R^{-1}\left(R u_{e}(t)+B^{T} P e(t)\right) \\
&-\left(\rho v(t)-\frac{1}{\rho} B^{T} P e(t)\right)^{T} \\
&\left.\times\left(\rho w(t)-\frac{1}{\rho} B^{T} P e(t)\right)\right] d t .
\end{aligned}
$$

From the game theory [11], [12], [22], we obtain the minimax control $u_{e}(t)$ as in (48) and the worst case $w^{*}(t)$ as in (50). Hence

$$
\begin{aligned}
& \min _{u_{e}(t)} \max _{w(t)} J\left(e, u_{e}, w\right) \\
& =e^{T}(0) \operatorname{Pe}(0)+\frac{1}{\gamma} \tilde{\Theta}^{T}(0) \tilde{\Theta}(0) \\
& \quad-e^{T}\left(t_{f}\right) \operatorname{Pe}\left(t_{f}\right)-\frac{1}{\gamma} \tilde{\Theta}^{T}\left(t_{f}\right) \tilde{\Theta}\left(t_{f}\right) \\
& \leq e^{T}(0) \operatorname{Pe}(0)+\frac{1}{\gamma} \tilde{\Theta}^{T}(0) \tilde{\Theta}(0) .
\end{aligned}
$$

The above inequality holds due to the fact, $P=P^{T}>0$, and $\gamma>0$. By the definition (45), the (55) is equivalent to the performance (43). Moreover, if $e(0)=0$ and $\widetilde{\Theta}(0)=0$, then (55) is reduced to (44).

Remark 5: $w^{*}(t)$ denotes a possible disturbance in $L_{2}\left[0, t_{f}\right]$, which makes the worst case influence on the tracking error $e(t)$ from the $L_{2}$-norm point of view.

Remarks 6:

1) In order to guarantee the positive definite solution $P$ of the algebraic Riccati-like equation (49), the following constraint must hold [11]:

$$
R^{-1}-\frac{1}{\rho^{2}} I>0
$$

or

$$
\rho^{2} I>R
$$

i.e., for a prescribed attenuation level $\rho$, the weighting matrix $R$ on the control $u_{e}(t)$ in (48) must satisfy the above constraint to guarantee the solvability of minimax tracking control of the uncertain wheeled vehicle system in (26). Hence, a robust tracking design with arbitrary attenuation of the influence of $w(t)$ is possible by the proposed method. However, if the attenuation level $\rho$ is specified to be a very small value, by the limit of (57), the singular value of $R^{-1}$ must be very large. In this situation, it will require large control energy. Therefore, there is a tradeoff between the attenuation level and the control energy.

2) Notice that the bounds on $\Theta$ and $e$ need not be known or be specified. Moreover, additional tools from the projec- tion algorithm [20] can be used to analyze the problem of the bound of $\Theta$. Assume that the constraint set $\Omega_{\Theta}$ is specified as $\Omega_{\Theta} \equiv\left\{\Theta \mid\|\Theta\| \leq M_{f}\right\}$, where $M_{f}$ is a positive constant. Then, the parameter update law in (47) must be modified as [20]

$$
\dot{\Theta}=\left\{\begin{array}{c}
\gamma \Xi^{T}(\eta) B^{T} P e(t), \\
\text { if }\|\Theta\|<M_{f} \text { or } \quad\left(\|\Theta\|=M_{f}\right. \\
\text { and } \left.\Theta^{T} \Xi^{T}(\eta) B^{T} P e(t)<0\right) \\
\gamma \Xi^{T}(\eta) B^{T} P e(t)-\gamma \frac{\Theta^{T} \Xi^{T}(\eta) B^{T} P e(t)}{\|\Theta\|^{2}} \Theta \\
\text { if }\|\Theta\| \geq M_{f} \text { and } \Theta^{T} \Xi^{T}(\eta) B^{T} P e(t) \geq 0
\end{array}\right.
$$

Since $\tilde{\Theta}^{T}(t)\left[\Xi^{T}(\eta) B^{T} P e(t)+(1 / \gamma) \dot{\tilde{\Theta}}(t)\right] \leq 0$ in $(52)$ due to the update law (58), the minimax tracking performance (43) can also be guaranteed.

3) The boundedness of $e(t)$ and $\eta(t)$ are discussed in the Appendix.

\section{Discussion:}

1) The proposed control scheme in this paper can deal with the following general nonlinear systems:

$$
\left(M_{0}+\Delta M\right) \ddot{q}+\left(C_{0}+\Delta C\right) \dot{q}+\left(G_{0}+\Delta G\right)=u+d
$$

with bounded uncertainties $\Delta M, \Delta C$, and $\Delta G$, and disturbance $d$ if the number of membership function is large enough.

2) There are two advantages of this approach over other fuzzy controllers:

a) The fuzzy controller in this paper is updated to approximate only the unmodeled term $f(\eta)$ in (32) rather than the true model terms, it products less approximation errors than the traditional fuzzy controller which is updated to approximate the whole model.

b) The proposed nonlinear controller (46) is a hierarchical control algorithm and is divided into three parts to achieve their design purposes, respectively. The first part contains $K_{1}$ and $K_{2}$, and are designed to achieve the asymptotical tracking of the nominal wheeled vehicle system. The second part, i.e., fuzzy controller $\Xi \Theta$ is tuned to cancel the uncertain part $f(\eta)$ as much as possible. Finally, the minimax control $u_{e}$ is designed to attenuate the effects of the residue of cancelation $f(\eta)-u_{e}$ as well as the external disturbance $d(t)$ on the tracking error.

3) In our design method, the fuzzy controller $\Xi \Theta$ is tuned to cancel the uncertain part $f(\eta)$ as much as possible. This resolves most problems on other control methods in which the bounds of the system uncertainties are needed to known and which are difficult to compute. On this viewpoint, the simulation results using the sliding mode control method are discussed in Section $\mathrm{V}$ to compare with our control method.

\section{DESIGN PROCEDURE}

Based on the above analysis, a design procedure for the fuzzybased minimax tracking control of wheeled vehicle systems can be outlined as follows: 


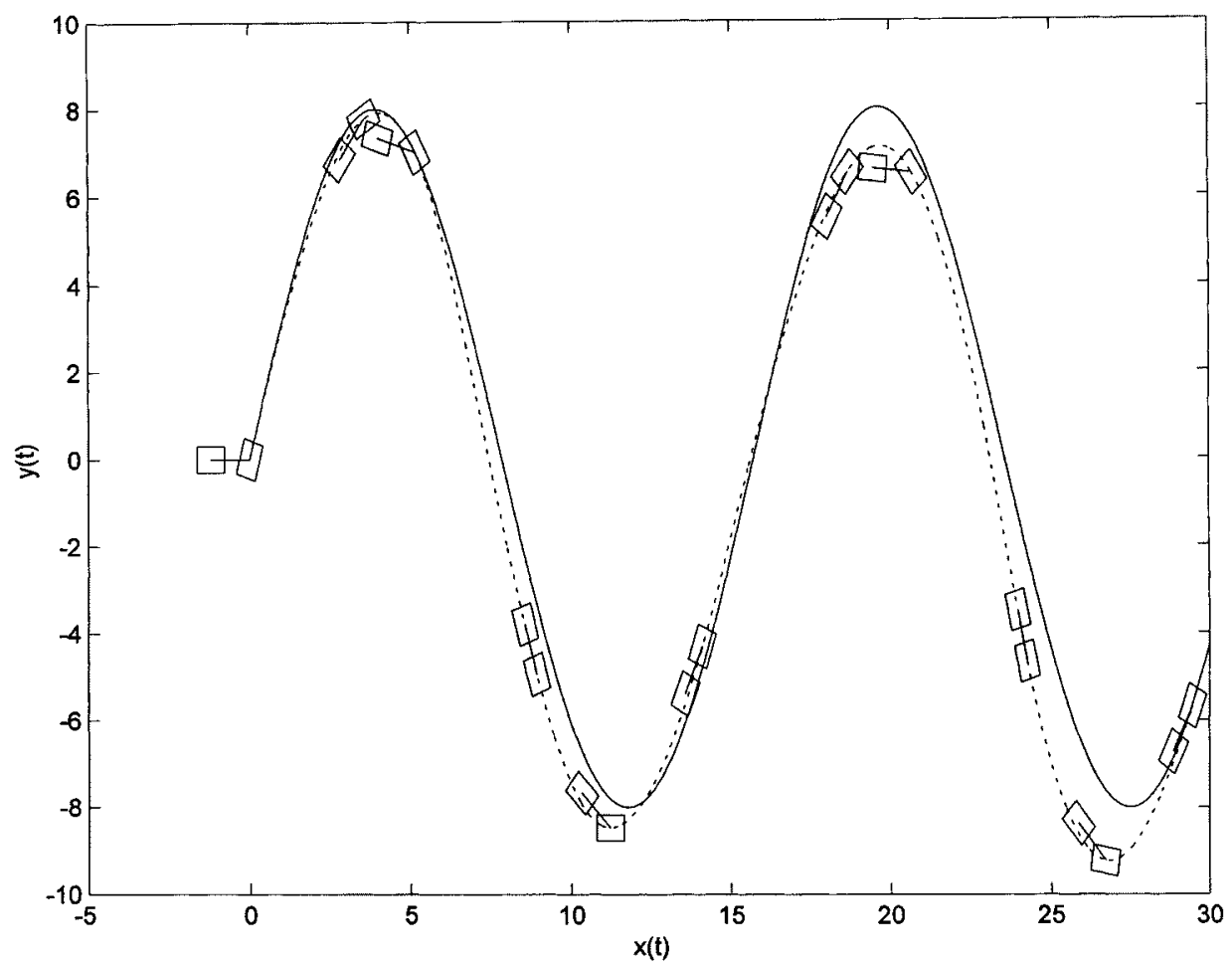

Fig. 3. Trajectory of the wheeled vehicle in X-Y plane when $\rho=0.5$.

Step 1) Specify $k_{11}, k_{12}, k_{21}$, and $k_{22}$ to determine matrix $A$ with desired eigenvalues.

Step 2) Decide the fuzzy architecture $\Xi(\eta)$ and specify the desired attenuation level $\rho$.

Step 3) Select positive-definite weighting matrices $Q$ and $R$ with $R<\rho^{2} I$ to guarantee the solvability of the minimax tracking.

Step 4) Solve the positive-definite matrix $P$ from the Riccati-like equation (49).

Step 5) Apply the control $u(t)$ in (46) to the uncertain wheeled vehicle system (26), by using the minimax tracking control law in (48) and the parameter update law (47).

In the next section, a design example is presented, given according to the above design procedure, to illustrate the performance of the proposed design method.

\section{SIMULATION EXPERIMENT}

In this section, the proposed fuzzy-based robust control on the tracking design of a wheeled vehicle is tested by using a computer. For the convenience of simulation, we consider a wheeled vehicle system as shown in Fig. 1, with the nominal parameters of this wheeled vehicle system as follows [6]:

$$
\begin{aligned}
I_{v} & =10\left(\mathrm{kgm}^{2}\right), \quad I_{w}=0.005\left(\mathrm{kgm}^{2}\right) \\
M_{1} & =200(\mathrm{~kg}), \quad M_{2}=100(\mathrm{~kg}) \\
l & =0.3(\mathrm{~m}), \quad c=0.05\left(\mathrm{kgm}^{2} / \mathrm{s}\right) \\
r & =0.1(\mathrm{~m}), \quad k=5, \quad L=1.2(\mathrm{~m}) .
\end{aligned}
$$

Suppose that the desired reference trajectories in the Cartesian coordinate are $x_{1 d}=t$ and $y_{1 d}=8 \sin (0.4 t)$. Assume the parameters $I_{v}, I_{w}, c, M_{1}$ and $M_{2}$ to be perturbed in the following form:

$$
\begin{aligned}
\Delta I_{v} & =0.1 \sin (t), \quad \Delta I_{w}=0.0001 \cos (t) \\
\Delta M_{1} & =2 \cos (t), \quad \Delta M_{2}=1.5 \cos (t)
\end{aligned}
$$

and

$$
\Delta c=0.001 \sin (t)
$$

respectively. Moreover, the exogenous disturbances $d_{1}^{\prime}$ and $d_{2}^{\prime}$ are sinusoidal waves with period $T_{p}=2 \pi$, i.e.,

$$
\begin{aligned}
& d_{1}^{\prime}=-0.3+0.3 \sin t \\
& d_{2}^{\prime}=0.2+0.2 \sin t
\end{aligned}
$$

Obviously, the parameter uncertainties and exogenous disturbances are significant. Therefore, the proposed fuzzy-based minimax tracking control algorithm is employed to treat this robust tracking control design. Now, following the design procedure as given in the above section, the robust tracking control design is achieved by the following steps.

Step 1) Specify $K_{1}=\left[\begin{array}{ll}5 & 0 \\ 0 & 5\end{array}\right], K_{2}=\left[\begin{array}{ll}5 & 0 \\ 0 & 5\end{array}\right]$ such that the eigenvalues of the nominal tracking system are $-1.3820,-3.6180,-1.3820$, and -3.6180 .

Step 2) Because there are two outputs of fuzzy logic system with each corresponding to seven fuzzy rules and four state variables. The following 56 membership functions (i.e., $M=7$ ) are selected:

$$
\begin{aligned}
& \mu_{F_{j}^{i 1}}=\exp \left[-\left(\left(\eta_{j}-3 \times a_{j}\right) / b_{j}\right)^{2}\right] \\
& \mu_{F_{j}^{i 2}}=\exp \left[-\left(\left(\eta_{j}-2 \times a_{j}\right) / b_{j}\right)^{2}\right] \\
& \mu_{F_{j}^{i 3}}=\exp \left[-\left(\left(\eta_{j}-1 \times a_{j}\right) / b_{j}\right)^{2}\right]
\end{aligned}
$$



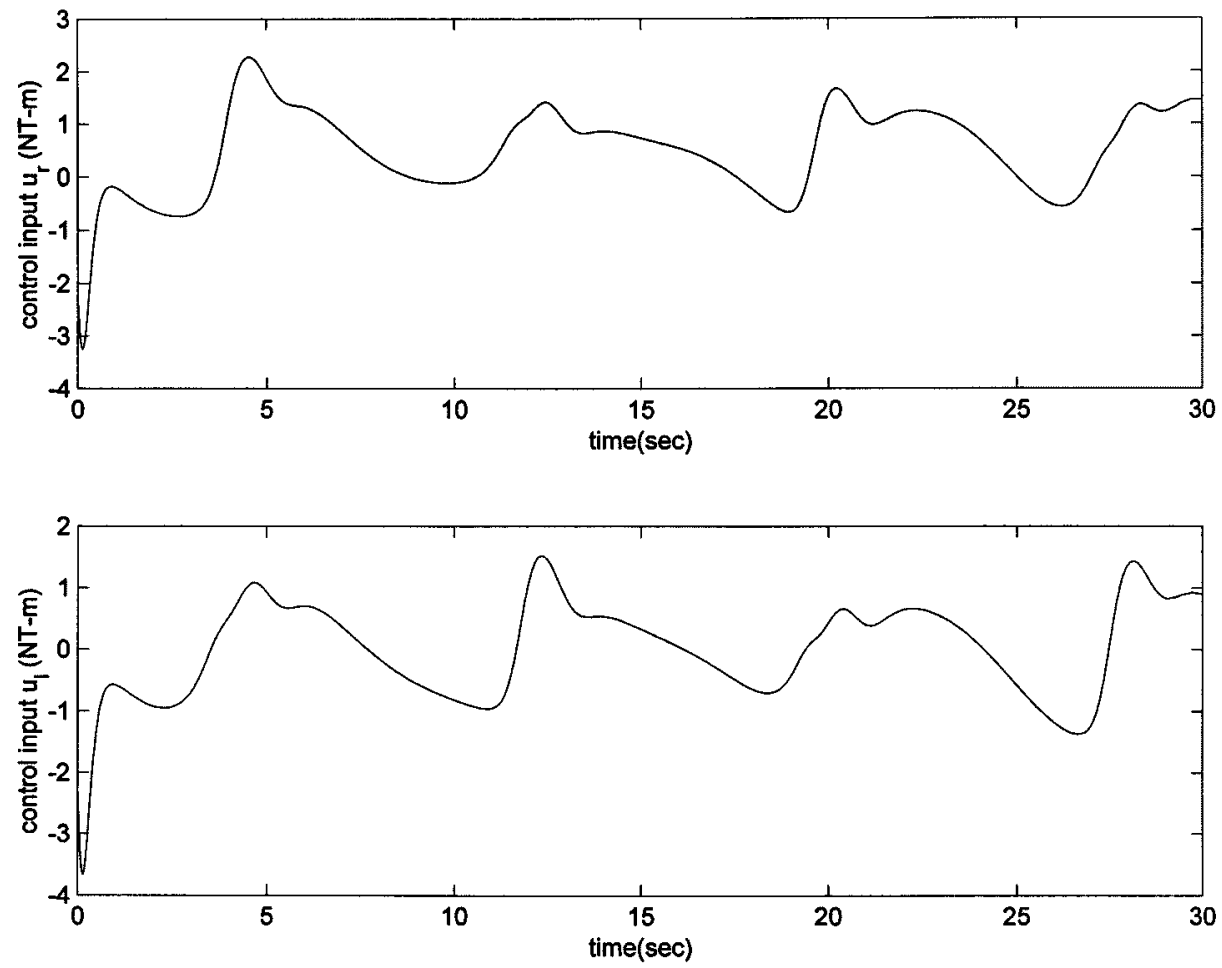

Fig. 4. Control inputs applied to the wheeled vehicle when $\rho=0.5$.

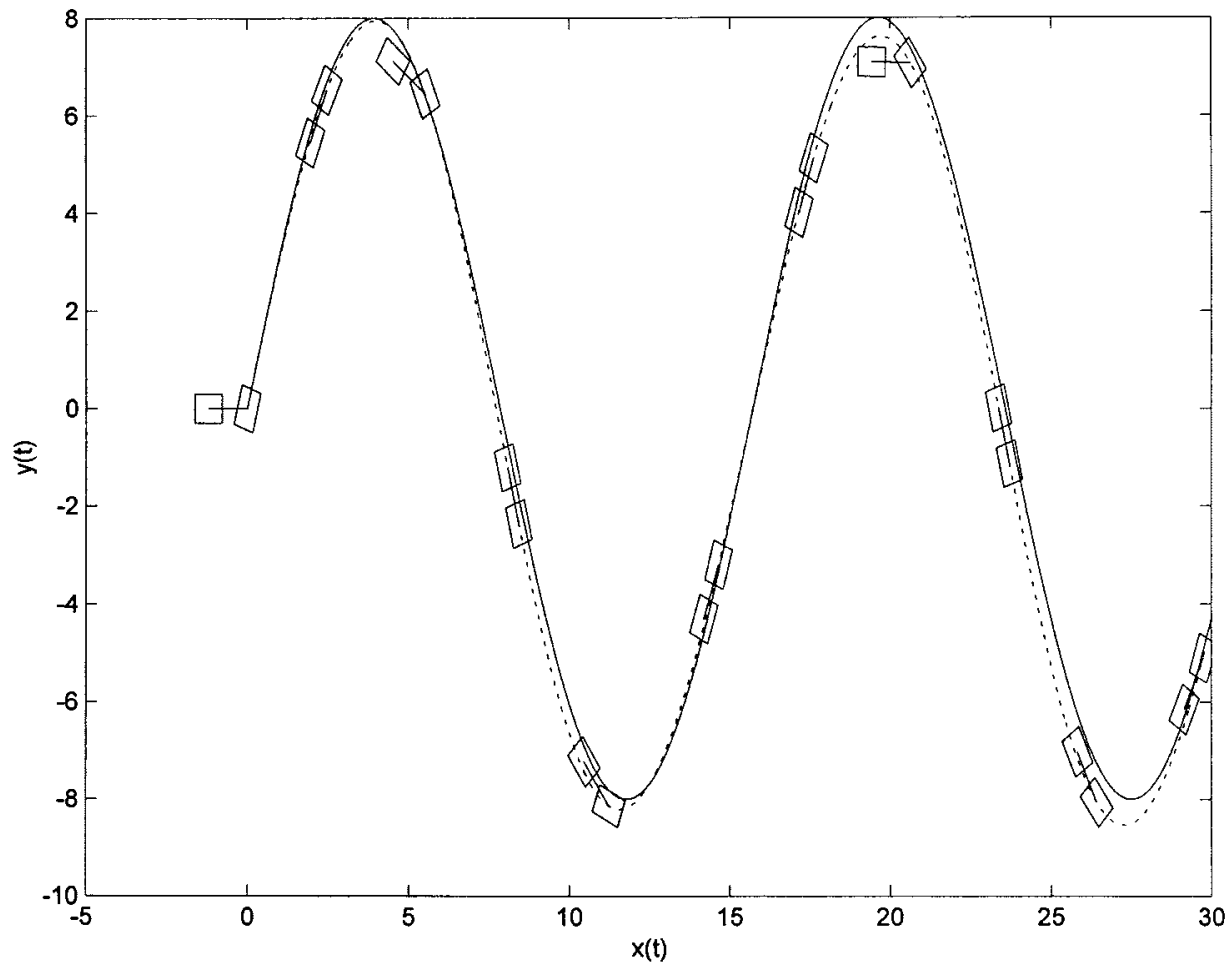

Fig. 5. Trajectory of the wheeled vehicle in X-Y plane when $\rho=0.2$.

$$
\begin{aligned}
& \mu_{F_{j}^{i 4}}=\exp \left[-\left(\eta_{j} / b_{j}\right)^{2}\right] \\
& \mu_{F_{j}^{i 5}}=\exp \left[-\left(\left(\eta_{j}+1 \times a_{j}\right) / b_{j}\right)^{2}\right] \\
& \mu_{F_{j}^{i 6}}=\exp \left[-\left(\left(\eta_{j}+2 \times a_{j}\right) / b_{j}\right)^{2}\right] \\
& \mu_{F_{j}^{i 7}}=\exp \left[-\left(\left(\eta_{j}+3 \times a_{j}\right) / b_{j}\right)^{2}\right]
\end{aligned}
$$

for $i=1,2$, and $j=1,2,3,4$ where $a_{1}=0.5$, $a_{2}=0.5, a_{3}=1, a_{4}=1, b_{1}=100, b_{2}=100$, $b_{3}=1000, b_{4}=1000$. The fuzzy rules in the following form are included in the fuzzy rule bases: $R^{(i 1)}:$ If $\eta_{1}$ is $F_{1}^{i 1}, \eta_{2}$ is $F_{2}^{i 1}, \eta_{3}$ is $F_{3}^{i 1}$ and $\eta_{4}$ is $F_{4}^{i 1}$, 

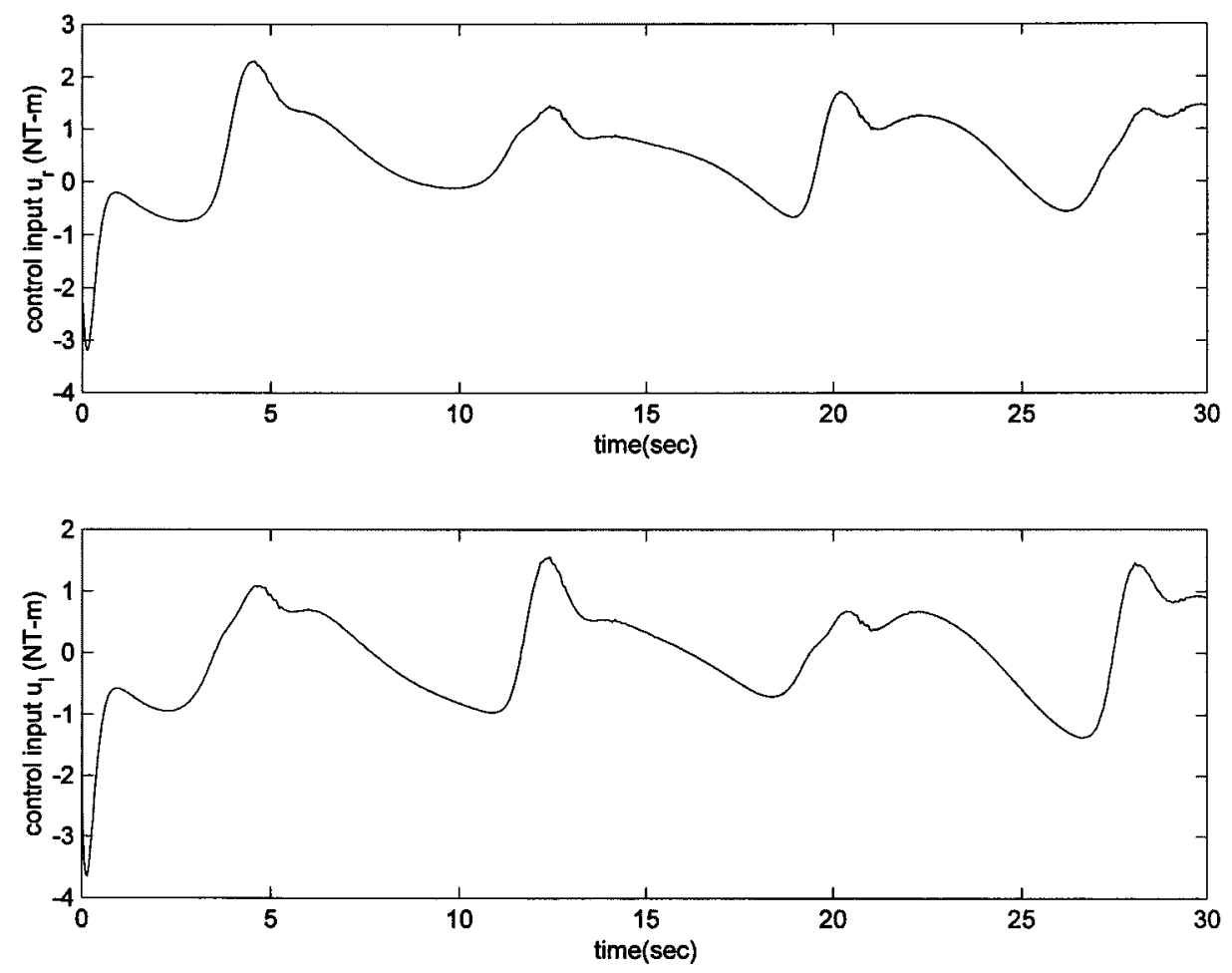

Fig. 6. Control inputs applied to the wheeled vehicle when $\rho=0.2$.

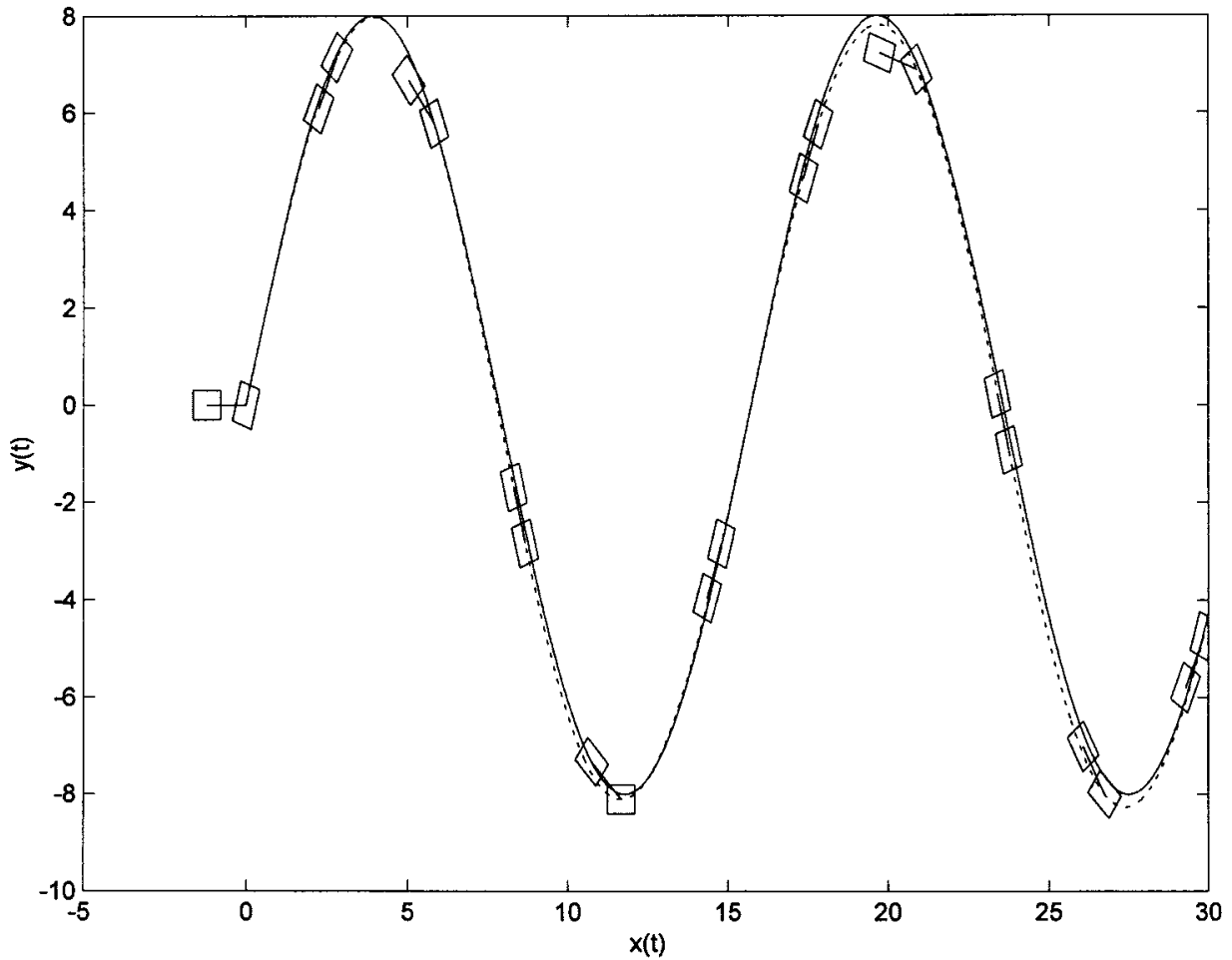

Fig. 7. Trajectory of the wheeled vehicle in X-Y plane when $\rho=0.1$.

Then $u_{f i}$ is $G^{i 1}$

$R^{(i 2)}$ : If $\eta_{1}$ is $F_{1}^{i 2}, \eta_{2}$ is $F_{2}^{i 2}, \eta_{3}$ is $F_{3}^{i 2}$ and $\eta_{4}$ is $F_{4}^{i 2}$,

Then $u_{f i}$ is $G^{i 2}$

$R^{(i 3)}:$ If $\eta_{1}$ is $F_{1}^{i 3}, \eta_{2}$ is $F_{2}^{i 3}, \eta_{3}$ is $F_{3}^{i 3}$ and $\eta_{4}$ is $F_{4}^{i 3}$,
Then $u_{f i}$ is $G^{i 3}$

$R^{(i 4)}:$ If $\eta_{1}$ is $F_{1}^{i 4}, \eta_{2}$ is $F_{2}^{i 4}, \eta_{3}$ is $F_{3}^{i 4}$ and $\eta_{4}$ is $F_{4}^{i 4}$, Then $u_{f i}$ is $G^{i 4}$

$R^{(i \check{5})}:$ If $\eta_{1}$ is $F_{1}^{i \check{5}}, \eta_{2}$ is $F_{2}^{i \check{5}}, \eta_{3}$ is $F_{3}^{i \check{5}}$ and $\eta_{4}$ is $F_{4}^{i \check{5}}$, 

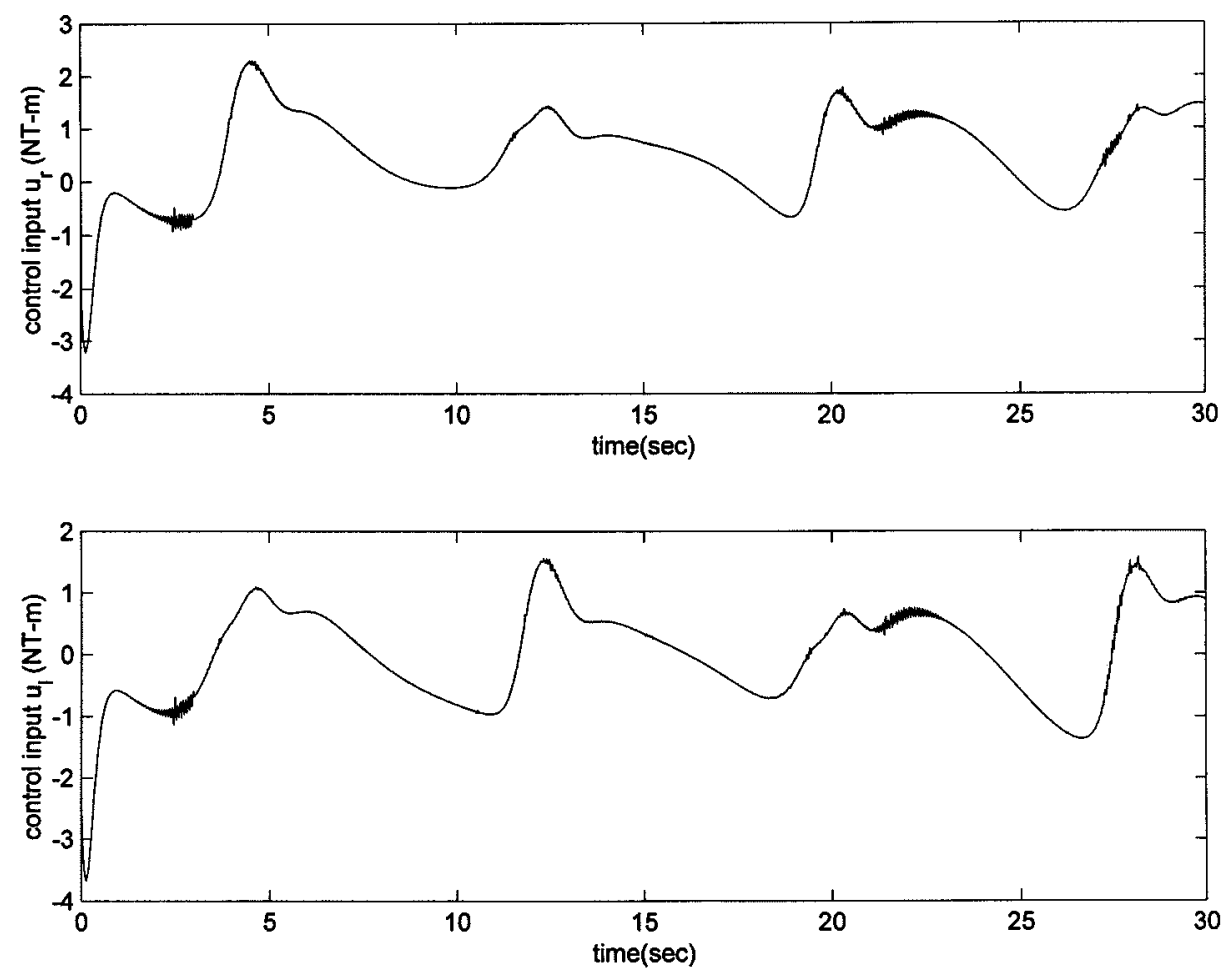

Fig. 8. Control inputs applied to the wheeled vehicle when $\rho=0.1$.

$$
\begin{aligned}
& \text { Then } u_{f i} \text { is } G^{i 5} \\
& R^{(i 6)} \text { : If } \eta_{1} \text { is } F_{1}^{i 6}, \eta_{2} \text { is } F_{2}^{i 6}, \eta_{3} \text { is } F_{3}^{i 6} \text { and } \eta_{4} \text { is } F_{4}^{i 6} \text {, } \\
& \text { Then } u_{f i} \text { is } G^{i 6} \\
& R^{(i 7)} \text { : If } \eta_{1} \text { is } F_{1}^{i 7}, \eta_{2} \text { is } F_{2}^{i 7}, \eta_{3} \text { is } F_{3}^{i 7} \text { and } \eta_{4} \text { is } F_{4}^{i 7} \text {, } \\
& \text { Then } u_{f i} \text { is } G^{i 7} \\
& \text { for } i=1,2 \text {. Denote } \\
& D=\sum_{k=1}^{7} \prod_{j=1}^{4} \mu_{F_{j}^{i k}}\left(\eta_{j}\right) \\
& \Theta_{1}=\left[\begin{array}{llll}
\theta_{11} & \theta_{12} & \cdots & \theta_{17}
\end{array}\right]^{T} \\
& \Theta_{2}=\left[\begin{array}{llll}
\theta_{21} & \theta_{22} & \cdots & \theta_{27}
\end{array}\right]^{T} \\
& \xi_{i}(\eta)=\left[\begin{array}{lll}
\left.\frac{\left(\prod_{j=1}^{4} \mu_{F_{j}^{i 1}}\right)}{D} \quad \ldots \quad \frac{\left(\prod_{j=1}^{4} \mu_{F_{j}^{i 7}}\right)}{D}\right] & \\
\text { for } i=1,2 .
\end{array}\right.
\end{aligned}
$$

The attenuation level $\rho$ is chosen to be $\rho=0.5$, $\rho=0.2$, and $\rho=0.1$, respectively.

Step 3) Select weighting matrix $Q=10 I$, and $R=0.25 \rho^{2} I$ to guarantee the solvability of the minimax tracking problem.
Step 4) Solve the Riccati-like equation (49). In case of $\rho=$ 0.5 , we have the solution

$$
P=\left[\begin{array}{cccc}
10.5732 & 0 & 0.5868 & 0 \\
0 & 10.5732 & 0 & 0.5868 \\
0.5868 & 0 & 0.6344 & 0 \\
0 & 0.5868 & 0 & 0.6344
\end{array}\right]
$$

In case of $\rho=0.2$, we have the solution

$$
P=\left[\begin{array}{cccc}
10.3002 & 0 & 0.3045 & 0 \\
0 & 10.3002 & 0 & 0.3045 \\
0.3045 & 0 & 0.3153 & 0 \\
0 & 0.3045 & 0 & 0.3153
\end{array}\right]
$$

In case of $\rho=0.1$, we have the solution

$$
P=\left[\begin{array}{cccc}
10.1653 & 0 & 0.1667 & 0 \\
0 & 10.1653 & 0 & 0.1667 \\
0.1667 & 0 & 0.1697 & 0 \\
0 & 0.1667 & 0 & 0.1697
\end{array}\right]
$$

In this simulation, the soft are WINDOWS MATLAB 5.0 is used to solve the algebraic Riccati equation.

Step 5) Use (46)-(48) to obtain the minimax tracking control $u_{e}(t)$, control input $u(t)$ and the adaptive law for $\Theta$, respectively. The simulation results are presented in Figs. 3-8. The position and applied torques of the wheeled vehicle are presented in Figs. 3 and 4 when $\rho=0.5$, Figs. 5 and 6 when $\rho=0.2$ and Figs. 7 and 8 when $\rho=0.1$. Fig. 9 shows the state errors of the wheeled vehicle. Fig. 10 shows the $H_{\infty}$ performances of the wheeled vehicle. Fig. 11 shows the fuzzy control approximation errors. From the simulation results of the above three cases, it can be seen that the smaller attenuation level $\rho$ may yield 

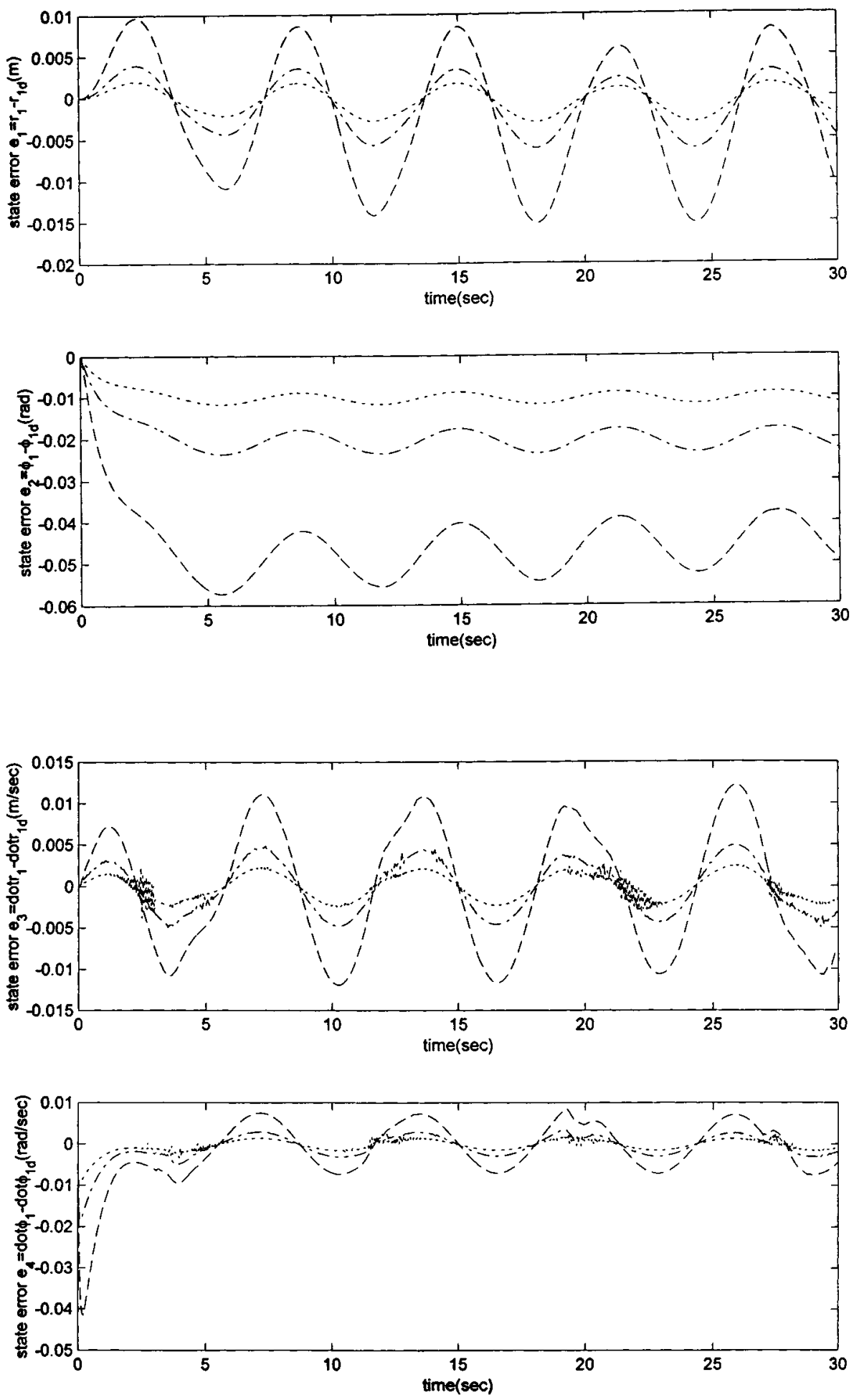

Fig. 9. State errors of the wheeled vehicle: “- - " " for $\rho=0.5$, “- .-." for $\rho=0.2$, and “...” for $\rho=0.1$.

better tracking performance. The effect of $w(t)$, including exogenous disturbances and approximation error due to adaptive fuzzy elimination, is evidently attenuated as $\rho$ decreases. But the control inputs also indicate a high-gain effect of the designed controller as $\rho$ decreases. In practical control engineering design, this effect due to plant uncertainties and ex- ogenous disturbances must be diminished, which will otherwise damage the tracking performance of the whole system. However, the attenuation level $\rho$ cannot be decreased without considering the increase in the control input. This is a tradeoff between the amplitude of control signal $u_{e}(t)$ and the tracking performance. 


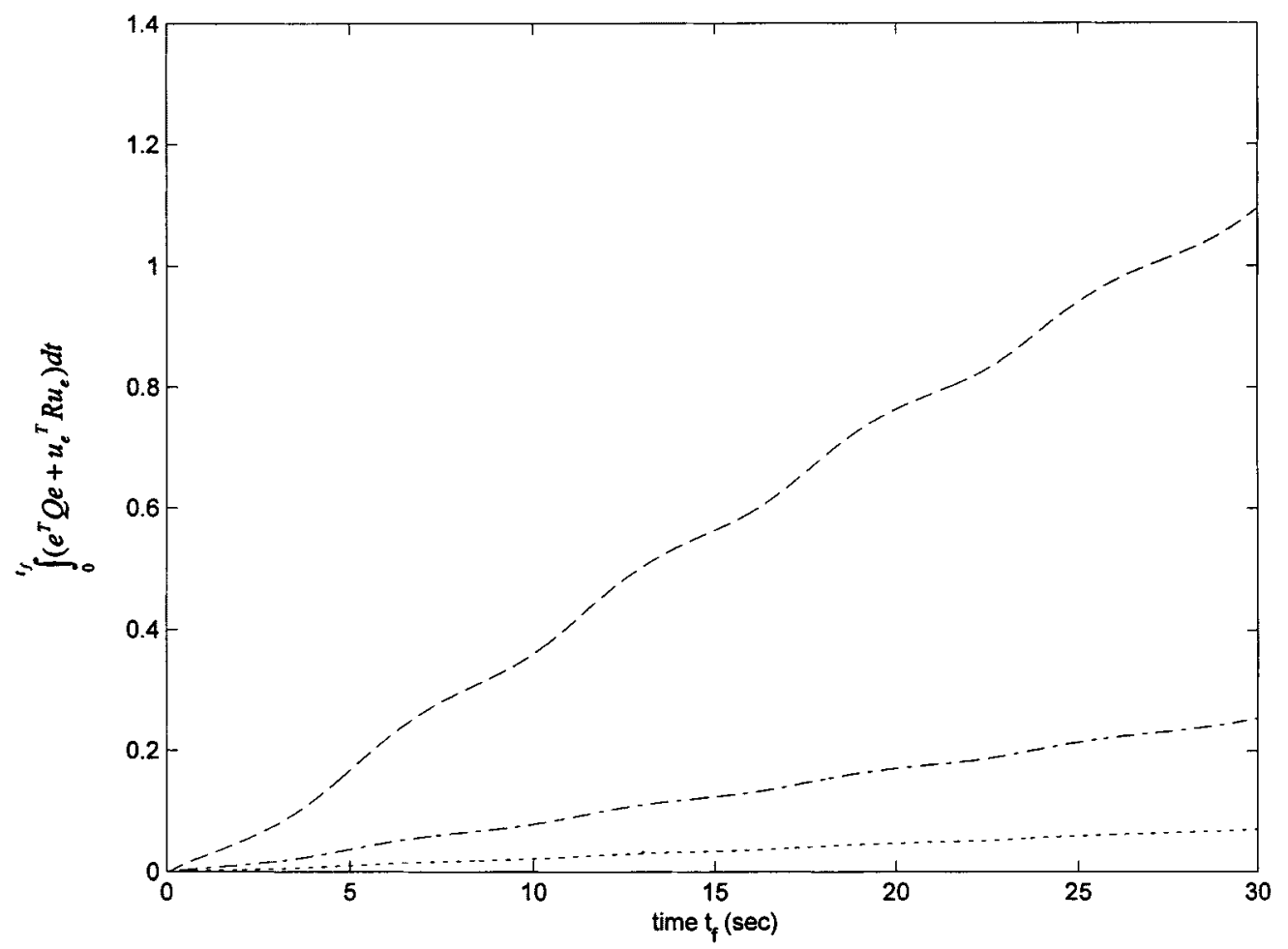

Fig. 10. $H_{\infty}$ performances of the wheeled vehicle: “- --” for $\rho=0.5$, “- .-.” for $\rho=0.2$, and “...” for $\rho=0.1$.
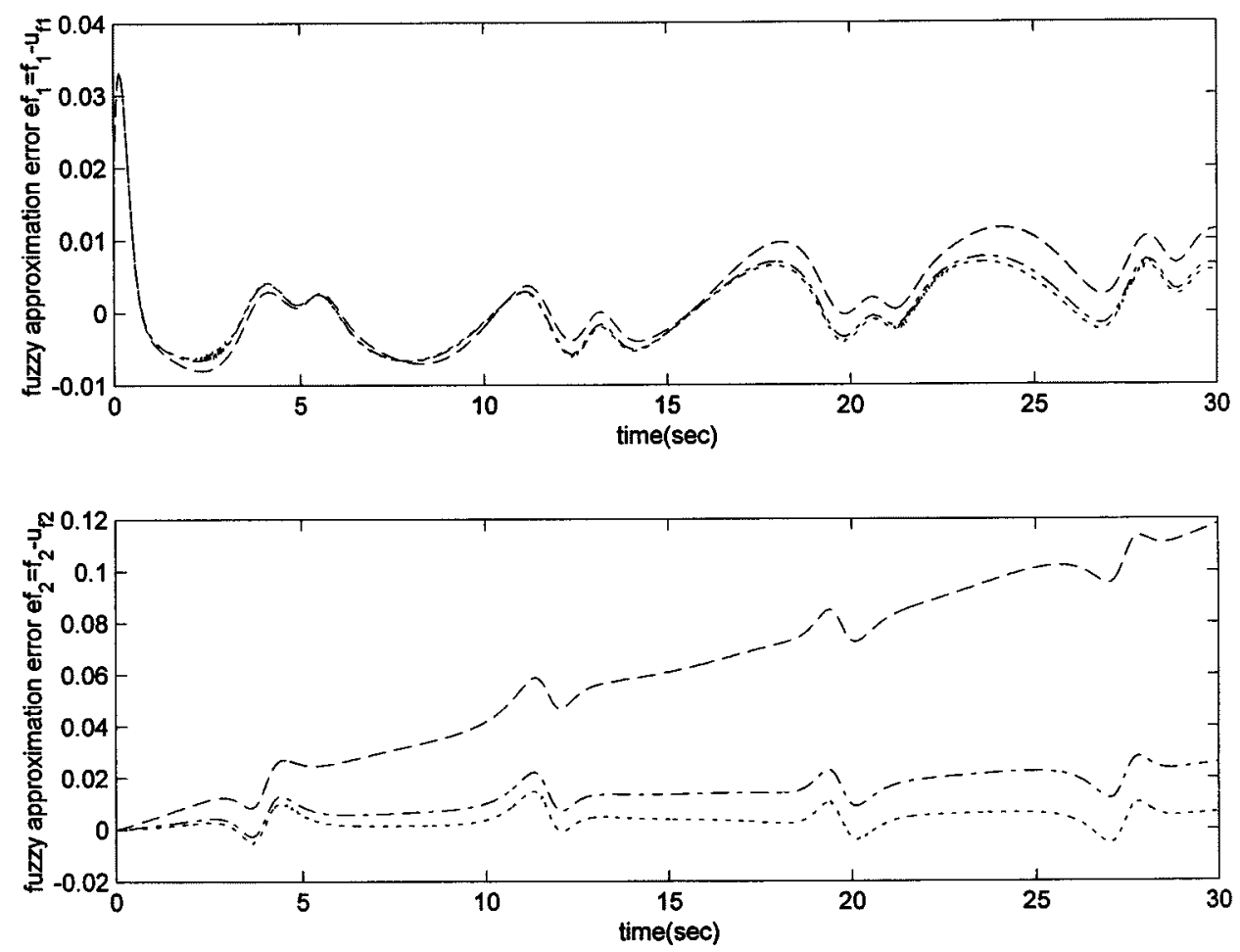

Fig. 11. Fuzzy control approximation errors: “- - -” for $\rho=0.5$, “- .-." for $\rho=0.2$, and “...” for $\rho=0.1$.

To compare with our design method, simulation results using sliding mode control on the same vehicle system are shown in
Figs. 12 and 13. From these results, more chattering exists in the control values and it causes larger tracking errors. Besides, 


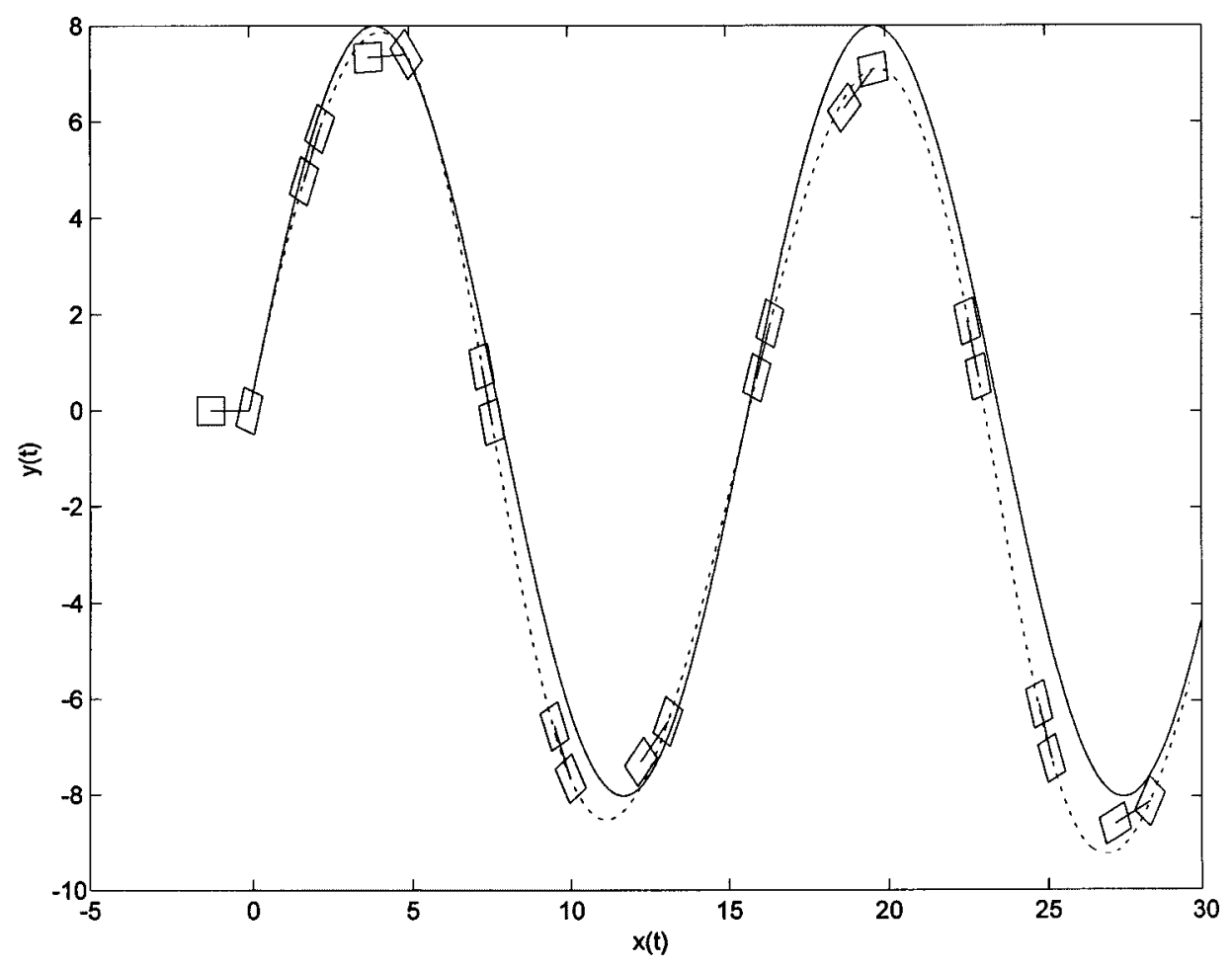

Fig. 12. Trajectory of the wheeled vehicle in X-Y plane using the sliding mode control method.
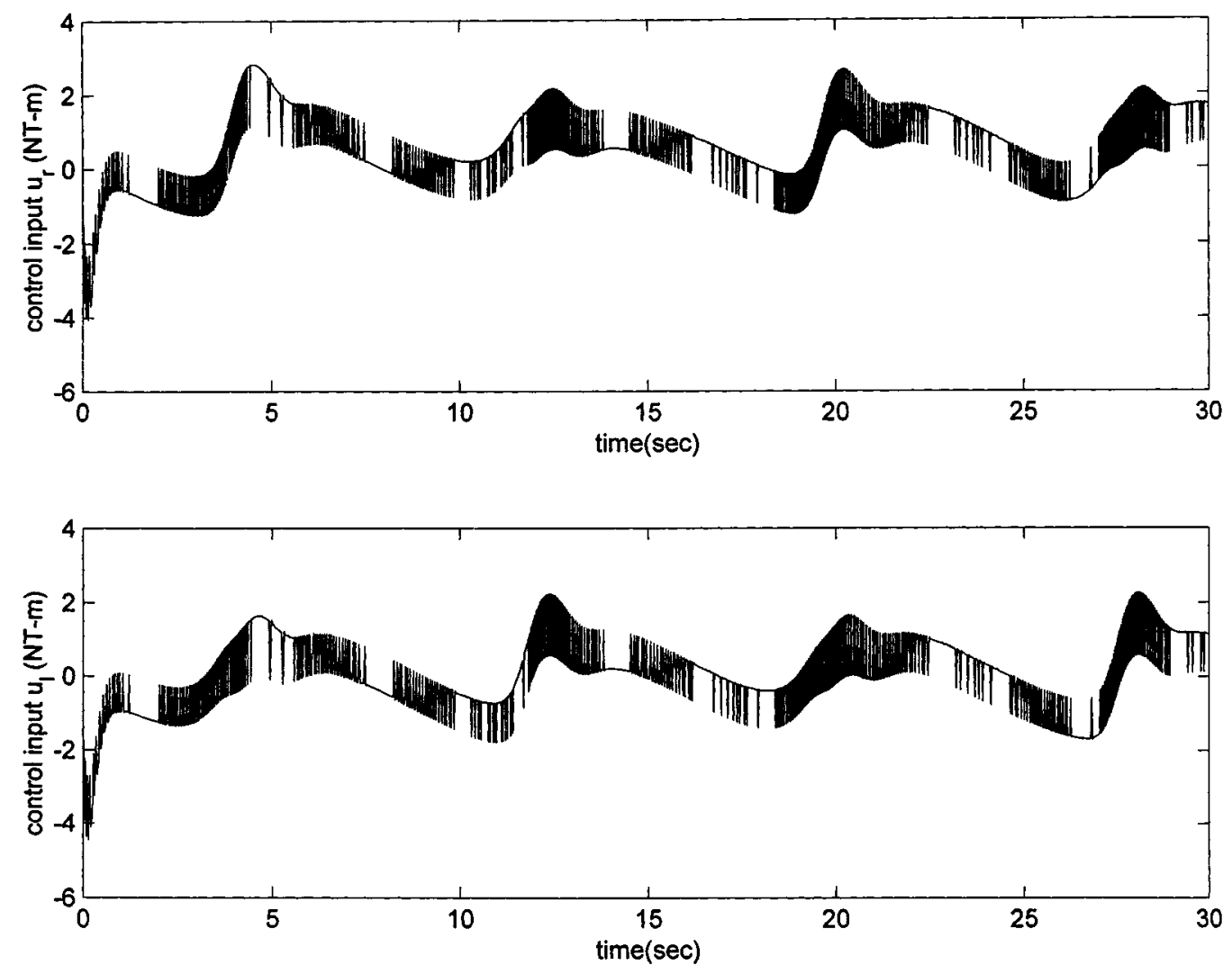

Fig. 13. Control inputs applied to the wheeled vehicle using the sliding mode control method. 
in the sliding mode control the bounds of the system uncertainties are needed to know and are difficult to get. By the control method designed in this paper, the the bounds of the system uncertainties are not needed and the control values are smoother and acceptable.

\section{CONCLUSION}

In this paper, an adaptive fuzzy technique and a minimax (or minimum $H_{\infty}$ ) attenuation technique are used for rough tuning and fine tuning, respectively, to treat the robust tracking control of uncertain wheeled vehicle systems. The enhancement of robust tracking performance is guaranteed by the proposed fuzzybased minimax control scheme from a more theoretical design perspective. Unlike the conventional nonlinear adaptive control, the uncertain dynamic need not be of linear parametrization in this work. Furthermore, the adaptive feedback linearization scheme is avoided and an inverse of adaptive matrix becomes unnecessary in our proposed control algorithm. The solvability of this robust minimax tracking problem is also illustrated. It has been observed that a desired minimax tracking performance can be achieved if weighting matrix $R$ on control signal is adequately specified.

Actually, the proposed fuzzy-based minimax tracking method can be applied to robust control design of any uncertain nonlinear nonholonomic constraint system. With the aid of adaptive fuzzy elimination algorithm, the minimax tracking control design can be extended from exactly known systems to uncertain systems. Furthermore, with the use of minimax attenuation technique, the tracking performance of the fuzzy-based control design for uncertain nonlinear systems can be significantly improved. Therefore, the proposed design algorithm is suitable for practical control design of uncertain wheeled vehicle systems. The proposed method is simple and the results are less conservative than the other methods. The simulation results clearly reveal that the robust tracking performance of uncertain wheeled vehicle systems can be enhanced by using the proposed method.

\section{APPENDIX}

\section{A. Boundedness of $e(t)$ and $\eta(t)$}

From (41) and (48), we get

$$
\begin{aligned}
\dot{e}(t) & =A e(t)+B u_{e}(t)+B \Xi^{T} \tilde{\Theta}(t)+B w(t) \\
& =A_{r} e(t)+B \Xi^{T} \tilde{\Theta}(t)+B w(t)
\end{aligned}
$$

where $A_{r}=A-B R^{-1} B^{T} P$. The solution of (59) can be obtained as

$$
e(t)=e^{A_{r} t} e(0)+\int_{0}^{t}\left[e^{A_{r}(t-\tau)}\left(B \Xi^{T} \tilde{\Theta}(\tau)+B w(\tau)\right)\right] d \tau
$$

By the asymptotical stability of $A_{r}$, we have

$$
\left\|e^{A_{r} t}\right\| \leq \beta \exp (-\alpha t)
$$

for some positive scales $\alpha$ and $\beta$, where $\|\cdot\|$ denotes the induced norm operator. Taking the norm operation on the both sides of (60), we obtain

$$
\begin{aligned}
\|e(t)\| \leq & \left\|e^{A_{r} t}\right\| \cdot\|e(0)\| \\
& +\int_{0}^{t}\left[\left\|e^{A_{r}(t-\tau)}\right\|\|B\|(\|\Xi\| \cdot\|\tilde{\Theta}(\tau)\|\right. \\
& +\|w(\tau)\|)] d \tau .
\end{aligned}
$$

By the inequality in (61), we get

$$
\begin{aligned}
\|e(t)\| \leq & \beta e^{-\alpha t}\|e(0)\|+\int_{0}^{t}\left[\beta e^{-\alpha(t-\tau)}\|B\|\right. \\
& \times(\|\Xi\| \cdot\|\tilde{\Theta}(\tau)\|+\|w(\tau)\|)] d \tau \\
\leq & \beta e^{-\alpha t}\|e(0)\| \\
& +\sup _{\tau \in[0, \infty]}[\|B\|(\|\Xi\| \cdot\|\tilde{\Theta}(\tau)\|+\|w(\tau)\|)] \\
& \times \int_{0}^{t}\left[\beta e^{-\alpha(t-\tau)}\right] d \tau \\
\leq & \beta e^{-\alpha t}\|e(0)\| \\
& +\sup _{\tau \in[0, \infty]}[\|B\|(\|\Xi\| \cdot\|\tilde{\Theta}(\tau)\|+\|w(\tau)\|)] \\
& \times\left[\frac{\beta}{\alpha}\left(1-e^{-\alpha t}\right)\right] .
\end{aligned}
$$

Then, the tracking error $e(t)$ is bounded by the following inequality:

$$
\begin{aligned}
\|e(t)\| \leq & \beta\|e(0)\|+\frac{\beta}{\alpha} \\
& \times \sup _{\tau \in[0, \infty]}[\|B\|(\|\Xi\| \cdot\|\tilde{\Theta}(\tau)\|+\|w(\tau)\|)] .
\end{aligned}
$$

for all $t$ since $\|\Xi\|$ and $\|\tilde{\Theta}(t)\|$ are bounded (see (39), (40), (42) and the projection algorithm (58)) and the norm $\|w(t)\|$ is assumed to be bounded also (see the Assumption 2). Furthermore, by the fact

$$
\eta=e+\eta_{d}
$$

and the bounded assumption of the reference trajectory $\eta_{d}$, the state vector $\eta$ is bounded.

\section{REFERENCES}

[1] G. J. Pappas and K. J. Kyriakopoulos, "Stabilization of nonholonomic vehicles under kinematic constraints," Int. J. Contr., no. 4, pp. 933-947, 1995.

[2] — , "Modeling and feedback control of nonholonomic mobile vehicles," in Proc. 31st Conf. Decision Contr., Tucson, AZ, 1992, pp. 2680-2685.

[3] - "Dynamic modeling and tracking control nonholonomic wheeled vehicles," in IFAC 12TH Triennial World Congr., Sydney, Australia, 1993, pp. 61-64.

[4] J.-P. Laumond, "Controllability of a multibody mobile robot," IEEE Trans. Robot. Automat., vol. 9, pp. 755-763, 1993.

[5] I. Kolmanovsky and N. H. Mcclamroch, "Developments in nonholonomic control problems," IEEE Trans. Contr. Syst. Technol., vol. 5, pp. 20-36, 1995.

[6] K. Watanable, J. Tang, M. Nakamura, S. Koga, and T. Fukuda, "A fuzzyGaussian neural network and its application to mobile robot control," IEEE Trans. Contr. Syst. Technol., vol. 4, pp. 193-199, 1996. 
[7] B. S. Chen, C. H. Lee, and Y. C. Chang, " $H_{\infty}$ tracking control design of uncertain nonlinear SISO systems: Adaptive fuzzy approach," IEEE Trans. Fuzzy Syst., vol. 4, pp. 32-43, 1996.

[8] X. J. Zeng and M. G. Singh, "Approximation theory of fuzzy systems-SISO case," IEEE Trans. Fuzzy Syst., vol. 2, pp. 162-176, 1994.

[9] B. Kosko, Neural Network and Fuzzy Systems. Englewood Cliffs, NJ: Prentice-Hall, 1992.

[10] T. Basar, $H_{\infty}$ Optimal Control and Related Minimax Design Problems: A Dynamic Game Approach. Boston, MA: Birkhauser, 1991.

[11] A. Stoorvogel, The $H_{\infty}$ Control Problem: A State Space Approach. Englewood Cliffs, NJ: Prentice-Hall, 1992.

[12] J. Doyle, K. Glover, P. P. Khargonekar, and B. A. Francis, "State-space solution to standard $H_{2}$ and $H_{\infty}$ control problems," IEEE Trans. Automat. Contr., vol. 34, pp. 831-847, 1989.

[13] B. S. Chen, T. S. Lee, and J. H. Feng, "A nonlinear $H_{\infty}$ control design in robotic systems under parameter perturbation and external disturbance," Int. J. Contr., vol. 59, no. 2, pp. 439-461, 1994.

[14] B. S. Chen, H. J. Uang, and C. S. Tseng, "Robust tracking enhancement of robot systems including motor dynamics: A fuzzy-based dynamic game approach," IEEE Trans. Fuzzy Syst., vol. 6, Nov. 1998.

[15] B. R. Barmish, M. Coreless, and G. Leitmann, "A new class of stabilizing controllers for uncertain dynamical systems," SIAM J. Contr. Optimization, vol. 21, pp. 246-255, 1983.

[16] M. Corless and G. Leitmann, "Continuous state feedback guaranteeing uniform ultimate boundedness of uncertain dynamic systems," IEEE Trans. Automat. Contr., vol. 26, pp. 1139-1144, 1981.

[17] F. Garofalo and G. Leitmann, "Guaranteeing ultimate boundedness and exponential rate of convergence for a class of nominally linear uncertain systems," ASME J. Dynamic Syst., Measurements Contr., vol. 111, pp. 584-588, 1989.

[18] D. Driankov, H. Hellendoorn, and M. Reinfrank, An Introduction to Fuzzy Control. Berlin, Germany: Springer-Verlag, 1993.

[19] M. Jamshidi, N. Vadiee, and T. J. Ress, Fuzzy Logic and Control. Englewood Cliffs, NJ: Prentice-Hall, 1993.

[20] K. S. Narendra and A. M. Annaswamy, Stable Adaptive Systems. Englewood Cliffs, NJ: Prentice-Hall, 1989.

[21] L. X. Wang, Adaptive Fuzzy Systems and Control: Design and Stability Analysis. Englewood Cliffs, NJ: Prentice-Hall, 1994.

[22] R. Isaacs, Differential Games. Huntington, NY: Kruger, 1975.

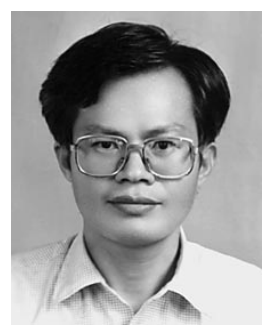

Bor-Sen Chen (M'82-SM'89) received the B.S. degree from Tatung Institute of Technology in 1970, the M.S. degree from National Central University, Taiwan, R.O.C., in 1973, and the Ph.D. degree from the University of Southern California, Los Angeles, in 1982.

He was a Lecturer, Associate Professor, and Professor at Tatung Institute of Technology from 1973 to 1987 . He is now a Professor at National Tsing Hua University, Hsin-Chu, Taiwan, R.O.C. signal processing.

His current research interests include control and

Dr. Chen has received the Distinguished Research Award from National Science Council of Taiwan four times. He is a Research Fellow of the National Science Council and the Chair of the Outstanding Scholarship Foundation.

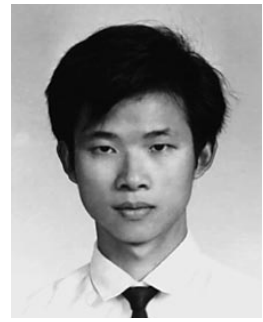

Charng-Shi Wu was born in Hsin Chu, Taiwan, in 1967. He received the B.S. degree from National Taiwan Institute of Technology, and the M.S. degree from National Tsing-Hua University, Taiwan, R.O.C., in 1992 and 1994, respectively, all in electrical engineering. $\mathrm{He}$ is currently working toward the Ph.D. degree in electrical engineering at National Tsing-Hua University.

His research interests include nonlinear systems, robust control, and spacecraft attitude control.

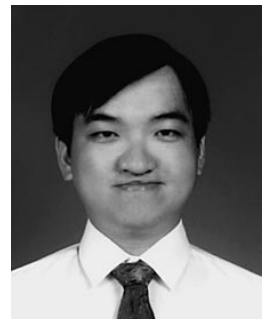

Huey-Jian Uang received the B.S. degree from the Department of Electrical Engineering, Feng-Chia University, Taichung, Taiwan, and the M.S. degree from National Tsing-Hua University, Hsin-Chu, Taiwan, in electrical engineering. He is currently working toward the Ph.D. degree in electrical engineering, National Tsing Hua University.

His current research interests include robotics, robust control, and fuzzy systems. 